\title{
DOMAIN-GENERAL AND DOMAIN-SPECIFIC BRAIN ACTIVATIONS AND NETWORKS IN VISUAL AND AUDITORY WORKING MEMORY
}

A Dissertation
presented to
The Faculty of the Graduate School
at the University of Missouri
In Partial Fulfillment
of the requirements for the Degree
Doctor of Philosophy
By
Dawei Li
Advisor: Dr. Nelson Cowan
DECEMBER, 2013


The undersigned, appointed by the dean of the Graduate School, have examined the dissertation entitled

DOMAIN-GENERAL AND DOMAIN-SPECIFIC BRAIN ACTIVATIONS AND NETWORKS IN VISUAL AND AUDITORY WORKING MEMORY

presented by Dawei Li,

a candidate for the degree of doctor of philosophy,

and hereby certify that, in their opinion, it is worthy of acceptance.

\begin{tabular}{c}
\hline Professor Nelson Cowan \\
\hline Professor Shawn Christ \\
\hline Professor Judith Goodman \\
Professor Jeff Johnson \\
\hline
\end{tabular}

Professor Jeff Rouder 


\section{ACKNOWLEDGEMENTS}

I would like to thank Professor Nelson, who introduced me into the field of cognitive psychology and guided me through my graduate study. I also would like to thank Professor Shawn Christ, who offered me a great deal of help on neuroimaging methods, Dr. Scott Saults, who helped me with behavioral methods for all three parts in this dissertation and produced the stimuli for Part III, Professor Jeff Johnson, who helped me with the multi-voxel pattern analysis in Part I, Professor Jeff Rouder, who taught me advanced statistical methods, and Professor Judith Goodman, who kindly served as the outside member in my committee. I also thank all the members in the Working Memory Lab at the University of Missouri for all their help during my graduate study. 


\section{TABLE OF CONTENTS}

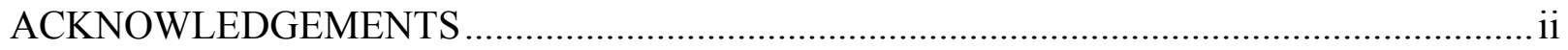

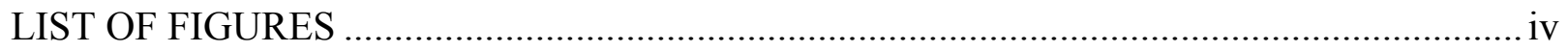

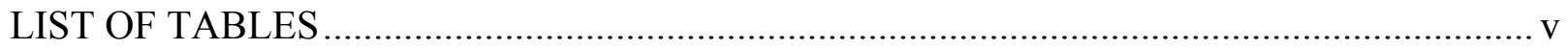

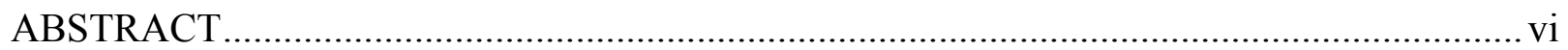

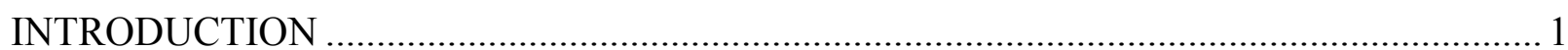

Behavioral Studies

Neuroscience Studies

PART I. PATTERN CLASSIFICATION IN THE LEFT INTRAPARIETAL SULCU ............ 9

Method

Results

Discussion

PART II. DOMAIN-GENERAL AND DOMAIN-SPECIFIC NEURAL NETWORKS

IN VISUAL AND AUDITORY WORKING MEMORY ............................................... 18

Method

Results

Discussion

PART III. NONVERBAL AUDITORY WORKING MEMORY ......................................... 46

Method

Results

Discussion

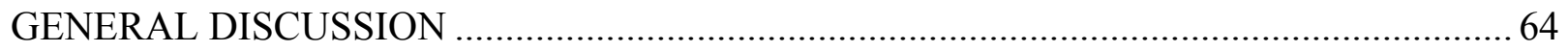

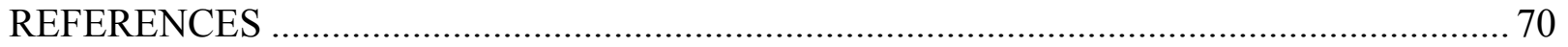

APPENDIX. Illustration of Constrained Principal Component Analysis .............................. 77

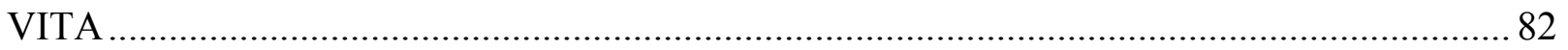




\section{LIST OF FIGURES}

Figure

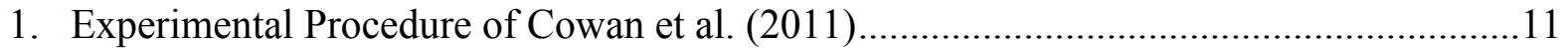

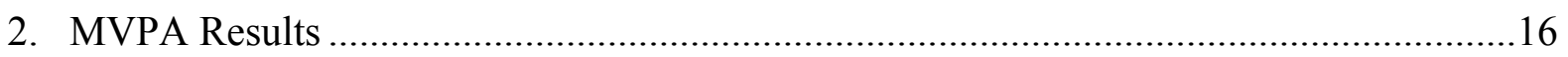

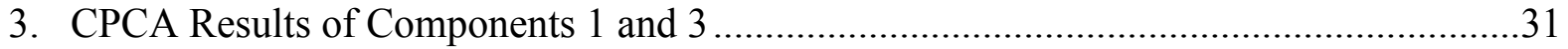

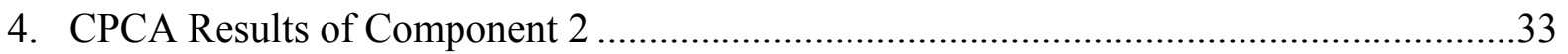

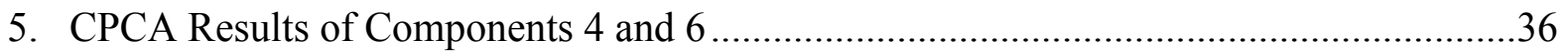

6. Significant Results of the Post-hoc Newman-Keuls Tests .............................................39

7. Experimental Procedure of the Nonverbal Auditory WM Study ..................................49

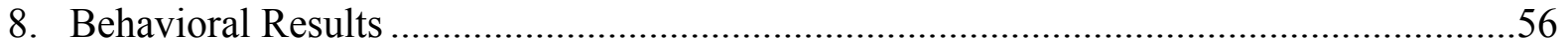

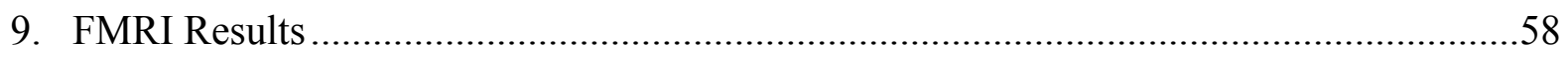

10. Time Course of the BOLD Signal Change in the Left Anterior IPS ROI ......................60 


\section{LIST OF TABLES}

Table

1. Brain regions included in Component 1 in the CPCA analysis ...................................26

2. Brain regions included in Component 3 in the CPCA analysis ....................................28

3. Brain regions included in Component 4 in the CPCA analysis ....................................29

4. Brain regions included in Component 6 in the CPCA analysis .......................................30 


\begin{abstract}
Working memory (WM) is a latent cognitive structure that involves active maintenance and manipulation of information for a short time. How items are stored in WM is an important issue that remains controversial. Whereas some researchers hold that different domains recruit different WM storage systems (domain-specific account), some other researchers argue that items of different domains share the same WM storage system (domain-general account).
\end{abstract}

The domain-specific and domain-general theories give rise to distinct predictions of brain activation patterns associated with WM storage. The domain-specific view predicts that different brain regions are involved in WM storage of stimuli from different domains. In contrast, the domain-general view predicts that a common brain region (or regions) is consistently involved in WM storage regardless of stimulus domains. Both predictions are supported by a few empirical findings in the previous literature, and therefore, the domainspecific versus domain-general argument remains unsettled.

This dissertation is aimed to provide further neuroscience evidence for the domaingeneral and domain-specific storage systems through the use of functional magnetic resonance imaging (fMRI). Specifically, this objective is pursued in three related parts.

Parts I and II are focused on two new analyses on a previous fMRI data set of visual and auditory WM. In the previous study, our group manipulated WM load to be either pure visual or auditory, or the combination of visual and auditory items in a single experiment. We found that a region in the left anterior intraparietal sulcus (IPS) was sensitive to both visual and auditory memory loads during WM maintenance (Cowan et al., 2011). Visual and auditory WM with the same level of memory load elicited the same level of activation in this brain region. The same activation level in the left anterior IPS, however, does not necessarily imply that the activation 
pattern in this brain region is the same across domains (e.g., Tamber-Rosenau et al., 2013). In Part I, using the same data set as Experiment 2 in Cowan et al. (2011), the activation pattern in the left anterior IPS was decoded with multivoxel pattern analysis (MVPA). The results showed that the activation pattern in the left anterior IPS was indistinguishable for visual and auditory domains when WM loads were the same across domains, which further supports the argument that a common region in the left anterior IPS supports both visual and auditory WM.

In Part II, an exploratory method, constrained principal component analysis (CPCA) was used to explore the domain-specific and domain-general neural networks involved in the same data set (Experiment 2 in Cowan et al., 2011). Analysis revealed evidence of both (1) specific neural networks responsive to either visual or auditory WM, and (2) general neural networks responsive to both visual and auditory WM. Importantly, only a general neural network, which includes a region of the left anterior IPS, was sensitive to WM load during the WM maintenance period, which supports the theory that items from different sensory domains are stored in a unitary WM storage system.

Parts I and II were focused on categorical visual and auditory stimuli. However, previous studies found that the estimates of WM capacity for nonverbal sounds were very different from those for categorical visual and auditory stimuli (Li, Cowan, \& Saults, 2013). To gain a comprehensive understanding of the brain mechanisms underlying WM maintenance, in Part III we studied the brain activities during a nonverbal auditory WM task. The participants were required to remember $2,3,4,5$, or 6 nonverbal sounds and to make a change-detection task after a short delay. The results revealed some evidence that different strategies were used for low and high memory loads and for different stimulus presentation methods. Importantly, the left anterior IPS did not show load-dependent activation across memory loads, indicating that the 
domain-general system might store abstract, categorical information which was difficult to extract from the nonverbal sounds used in this study.

Combining results from all three approaches, the left anterior IPS appears to be part of a neural network for maintenance of abstract, categorical information across stimulus domains. This characteristic of the left anterior IPS supports the existence of a domain-general system for WM storage which stores categorical information. 


\section{INTRODUCTION}

Working memory (WM) is a latent cognitive structure that involves active maintenance and manipulation of information for a short time (Baddeley, 1986; Cowan, 1995). WM is critical to the higher order cognitive functions such as language, planning, and decision making. How items are stored in WM, however, remains a debated issue. Some researchers hold that items from different sensory domains, such as vision and audition, are stored in relatively separate WM stores (Baddeley \& Hitch, 1974; Cocchini et al., 2002). This account is referred to as a domain-specific view of WM storage. In contrast, some researchers argue that stimuli from different sensory domains are stored in a unitary WM storage system (Cowan, 1995; Kane et al., 2004; Saults \& Cowan, 2007). This account is referred to as a domain-general view of WM storage.

The domain-specific and domain-general views give rise to distinct predictions of brain activity patterns underlying WM storage. For example, the domain-specific view predicts that different brain regions are involved in WM storage of stimuli from different domains, whereas the domain-general view predicts that a common brain region (or regions) would be consistently involved in WM storage regardless of sensory domains.

In this study, the mechanism of WM storage is examined using fMRI by investigating the domain-general and domain-specific brain activations in visual, verbal, and nonverbal auditory WM tasks.

In the following introduction sections, I will present the existing contributions from behavioral and neuroscience studies on the issue of WM storage. 


\section{Behavioral Studies}

Some researchers proposed that WM storage is domain-specific: items in different modalities, such as visual and auditory stimuli, are maintained in relatively separate WM stores. Baddeley and Hitch (1974) introduced a multi-component model of WM, proposing that WM can be divided into a "central executive system", and two "slave systems", phonological loop and visuospatial sketchpad. The central executive system is responsible for integration, coordination, and manipulation of the information held in the slave systems, and the slave systems are responsible for the temporary retention of phonological and visuospatial information, respectively. The phonological loop and visuospatial sketchpad have separate storage mechanisms and are independent from each other. This is a domain-specific account of WM storage.

The domain-specific account is supported by some behavioral studies. In a dualtask study, Cocchini and colleagues instructed the participants to concurrently perform pairwise combinations of a verbal WM task, a visual WM task, and perceptuomotor tracking (Cocchini et al., 2002). The result showed no impact of the verbal WM task on the visual WM task, indicating that the items in different modalities are maintained separately.

Some other studies, however, indicates that different sensory domains might share a common storage system in WM. Morey and Cowan conducted a WM experiment similar to the one by Cocchini and colleagues (Morey \& Cowan, 2004). The participants were instructed to memorize the colors of several visual squares (pure visual task). During some trials, they also needed to overtly repeat seven random digits presented at 
the beginning of the trial (combined task). The performance significantly decreased in the combined task compared with the pure visual task, indicating that visual and verbal WM might share the same storage resource. The authors suggest that the divergent results of Morey and Cowan (2004) and Cocchini et al. (2002) might be due to the slight difference between the experimental designs. Whereas the participants were required to overtly rehearse the verbal stimuli in Morey and Cowan (2004), the participants in Cocchini et al. (2002) only needed to rehearse the verbal stimuli covertly, which might have made the verbal task much easier and imposed less effect on the visual WM task. Moreover, Cocchini et al. (2002) did obtain a smaller, although not significant effect on visual WM imposed by the verbal task, indicating that verbal task with silent rehearsal might still to some extent affect visual WM performance.

To further study the interaction between visual and verbal WM, Morey and Cowan (2005) directly compared the effect of covert and overt rehearsal of verbal materials on visual WM task. In some trials, the participants were required to articulate aloud seven random digits during the maintenance period of a visual WM task; in some other trials, the subjects did not articulate, but silently rehearsed seven random digits when performing the same visual WM task. Performance in the silent rehearsal trials was not significantly different from the pure visual WM trials, but performance in the aloud articulation trials significantly decreased, indicating that overt rehearsal of a verbal WM load affects visual WM performance. Moreover, the previous study by Morey and Cowan (2004) revealed that overt articulation of the participant's own seven-digit telephone number during visual WM maintenance did not influence visual WM performance. These results suggest that only explicit retrieval of novel verbal items that 
is attention demanding affects visual WM, and that neither silent retrieval of novel verbal items nor overt retrieval of familiar verbal items impairs visual WM. The authors argue that visual maintenance and verbal retrieval share the same cognitive resource, which is most likely to be the focus of attention. This argument is also supported by the finding that when the visual load was as high as eight colored squares, visual WM performance was also impaired in the silent rehearsal trials, probably because visual WM took so much attention resource that even a small amount of attention taken by silent rehearsal could affect visual WM performance (Morey \& Cowan, 2005).

In a more recent study, Morey and Bieler (2013) showed that even at a low visual WM load, interference from a concurrent nonvisual WM task could still be observed. The participants were instructed to remember 2,3, or 4 visual objects composed of either pure features (colors/shapes), or bounds of features (colors and shapes). In some of the trials, they also needed to remember a tone presented right after the presentation of the visual objects, and to judge whether a second tone presented 1,200-ms after the first tone was the same or different as the first one. The analysis showed that the concurrent tone task impaired visual WM performance even at a low visual WM load of two, regardless of whether the visual objects were pure features or bounds of features. This result further supports the view that visual WM share the same storage resource with nonvisual WM.

Saults and Cowan (2007) investigated the property of WM storage with a similar dual-task paradigm as the one used by Cocchini et al. (2002) and Morey \& Cowan (2004). The participants were shown a combination of a visual square array and an auditory digit array and were required to remember only the visual array, only the auditory array, or both arrays. Five studies were conducted, three of which included both 
visual and auditory masks right after the stimuli presentation, and the other two did not. The visual mask was composed of an array identical to the initial visual array, except that each square was cut into seven horizontal stripes of the seven colors used in the experiments arranged in random order; the auditory mask consisted of simultaneous combination of the nine digits used in the experiments. The purpose of applying the masks was to eliminate the domain-specific sensory memory. The studies with masks yielded significant interference between modalities: when remembering both modalities, performance on visual and auditory arrays both decreased compared with when remembering only visual or only auditory items. Furthermore, the total number of visual and auditory items remembered was close to and no more than any of the capacities calculated from the pure visual or pure verbal conditions. The interference between visual and auditory WM supports a central multimodal storage system with a fixed capacity limit.

In a different approach, Kane and colleagues (2004) tested verbal and visuospatial stimuli in both short-term memory and WM tasks. The short-term memory tasks involved pure storage, and the WM tasks involved not only storage but also maintenance of verbal or visuospatial stimuli. Confirmatory factor analyses showed that the WM tasks were dominated by a domain-general factor, and that the short-term memory tasks were more domain-specific. Nevertheless, even the short-term memory tasks showed substantial correlation between verbal and visuospatial stimuli, suggesting that verbal and visuospatial WM not only have domain-specific storage systems but also share a domaingeneral storage system. 
Based on these findings, some researchers proposed a domain-general account that items in different sensory domains share a unitary WM storage system (Cowan, 1995; Kane et al., 2004). Notably, the domain-general account does not deny the existence of domain-specific storage systems. It only conflicts with the strong domainspecific account that stimuli of different domains can be completely dissociated from each other in WM storage. The debate on the characteristics of WM storage remains active in the field of cognitive psychology.

\section{Neuroscience Studies}

The neural mechanism of WM storage has been extensive studied in both human and nonhuman primate samples. Many studies found that the fronto-parietal network is consistently activated during WM (Owen et al., 2005; Rottschy et al., 2012). Several studies further showed that the posterior parietal cortex is involved in WM storage and that the prefrontal region is responsible for the top-down control of posterior brain regions (D'Esposito et al., 2006; Postle et al., 2006).

It is unclear, however, how items from different sensory domains are coded in the brain during WM tasks. The domain-specific view of WM storage predicts that visual and auditory stimuli are stored in spatially distinct brain regions. Consistent with this view, Smith and Jonides (1997) found lateralized activation of the left and right prefrontal cortex during maintenance of verbal versus spatial information, respectively. Additional support comes from studies suggesting that the dorsolateral prefrontal cortex is involved in spatial WM maintenance whereas the ventrolateral prefrontal cortex is 
involved in nonspatial object WM maintenance (Courtney et al., 1996; Haxby et al., 1994; Ungerleider et al., 1998).

In contrast to the domain-specific view, the domain-general view predicts that visual and auditory stimuli are stored in a common neural network, though secondary domain-specific mechanisms also may exist according to most versions of the theory. Evidence consistent with this view comes from our recent study (Cowan et al., 2011), which found that a region in the left IPS was consistently activated during the storage of stimuli in WM regardless of whether it was visual or verbal in nature. Overlapping brain regions have also been found to activate for verbal and spatial WM (Chein et al., 2011), visual and verbal WM (Majerus et al, 2010) as well as verbal and tonal WM (Koelsch et al., 2009).

As detailed above, previous neuroimaging studies did not fully resolve the domainspecific versus domain-general debate regarding the nature of WM storage. This is partly due to several limitations of these studies. First, until recent, most studies have focused on the activation levels in brain regions. The same activation level in a brain region across domains, however, does not necessarily imply the same activation pattern across domains. It is instead possible that two sensory domains recruit two distinct neuron assemblies intermingled in a single brain region. In this case, this region could show the same activation level for these two sensory domains, although it has different activation patterns and is not a domain-general region. Therefore, to conclude a domain-general region, activation pattern in this region must also be assessed. 
Second, the majority of the previous studies have relied on univariate statistical approaches. Univariate approaches, such as the general linear model, are focused on the time series of each voxel independently and disregard the correlation between voxels. It has been argued, however, that the correlation between spatially distant brain voxels is a critical feature of fMRI data sets and reflects important information about the neural networks underlying cognitive tasks, which would not otherwise be evident using a univariate approach alone. The multivariate approaches take into account such intervoxel correlations and thus could provide a more comprehensive view of the neural networks underlying WM.

Third, most of the previous studies used categorical stimuli, such as letters, digits, and colors with easily distinguishable hues. Categorical stimulus could be represented in an abstract form regardless of its sensory domain. For example, both the auditory word "red" and the visual color "red" could be represented by the concept of "redness" which does not imply any sensory feature. It is therefore likely that the abstract, categorical form of item representation is stored in the domain-general storage system in WM, and that the stimulus details are stored in the domain-specific storage systems. This hypothesis, however, is difficult to prove using solely categorical stimuli.

The current study aims to address these limitations and resolve the domain-specific versus domain-general issue via three parts. Parts I and II are focused on two new analyses of a previous data set on visual and auditory WM (Experiment 2 in Cowan et al., 2011) and address the first and the second limitations, respectively. Part III is focused on a new study on nonverbal auditory WM and addresses the third limitation. These three parts will be presented in separate sections in the following text. 


\section{PART I. PATTERN CLASSIFICATION IN THE LEFT INTRAPARIETAL SULCUS}

The intraparietal sulcus (IPS) in the parietal lobe has proved to be a critical brain region for WM storage in many studies. Todd and Marois (2004) manipulated visual WM load to be $1,2,3,4,6$, or 8 in a change-detection fMRI experiment. They found that the activity in bilateral IPS was associated with the number of visual items kept in WM, indicating that the IPS is responsible for visual WM storage. Our previous study (Cowan et al., 2011) used pure visual, pure auditory, and combinations of visual and auditory items in a single change-detection fMRI experiment. The results showed that a region in the left anterior IPS showed the same activation level for a certain memory load regardless of stimulus domains, and that it was the only region sensitive to both visual and auditory WM loads, suggesting that the left IPS is responsible for not visual-only but domain-general storage.

Our previous study (Cowan et al., 2011), however, was focused on solely the activation levels. It is still possible that even if the activation levels in the left anterior IPS are the same for visual and auditory WM, the activation patterns are different (Tamber-Rosenau et al., 2013). For example, if visual and auditory WM activate two distinct neuron assemblies intermingled in the left anterior IPS, they will invoke the same activation level but still have different activation patterns in this region. This scenario is quite possible given the large number of neurons in even a small region like the left anterior IPS. The human cerebral cortex has approximately 16 billion neurons, and it has about $25,3003 \times 3 \times 3 \mathrm{~mm}^{3}$ voxels (Lent et al., 2012). Therefore, a single $3 \times 3 \times 3 \mathrm{~mm}^{3}$ 
voxel includes 16 billion / 25, 300 $\approx 630,000$ neurons, and the left anterior IPS region in Cowan et al. (2011) includes about $373 \times 3 \times 3 \mathrm{~mm}^{3}$ voxels. It is possible that the large amounts of neurons in this region form distinct neuron assemblies, which, due to the spatial resolution and inherent physiological limitations of BOLD fMRI, cannot be distinguished through the examination of activation levels.

One method to investigate the activation patterns, multivoxel pattern analysis (MVPA), has been developed in recent years (Norman et al., 2006; Polyn et al., 2005). MVPA is a machine learning approach and can be used to train pattern classifiers to associate activation patterns in a brain region to cognitive states such as visual and auditory WM storage, and to make predictions of cognitive states based on performance of the classifiers. If the predictions behave significantly above chance, then this brain region carries distinct information for different cognitive states.

In this study, MVPA is applied to the Experiment 2 data set in Cowan et al. (2011) to decode the activation patterns in the left anterior IPS. In this experiment, the participants were instructed to remember either pure visual or pure auditory items, or combinations of visual and auditory items, for several seconds in a change-detection task. If the activation patterns are the same for visual and auditory WM with the same load, we will be more confident to conclude that this region is responsible for domain-general storage; if the activation patterns are different for visual and auditory WM, then this region carries domain-specific information and thus is not an area for domain-general storage. 


\section{Method}

For this study, data from Experiment 2 in Cowan et al. (2011) was analyzed with MVPA.

Detailed descriptions of the experimental procedure can be found in Cowan et al. (2011).

A brief summary of the experimental tasks is presented below.

Participants. Fifteen participants ( 7 male), ranging from 18 to 20 years old, were included in the analysis. All participants were college students at the University of Missouri.

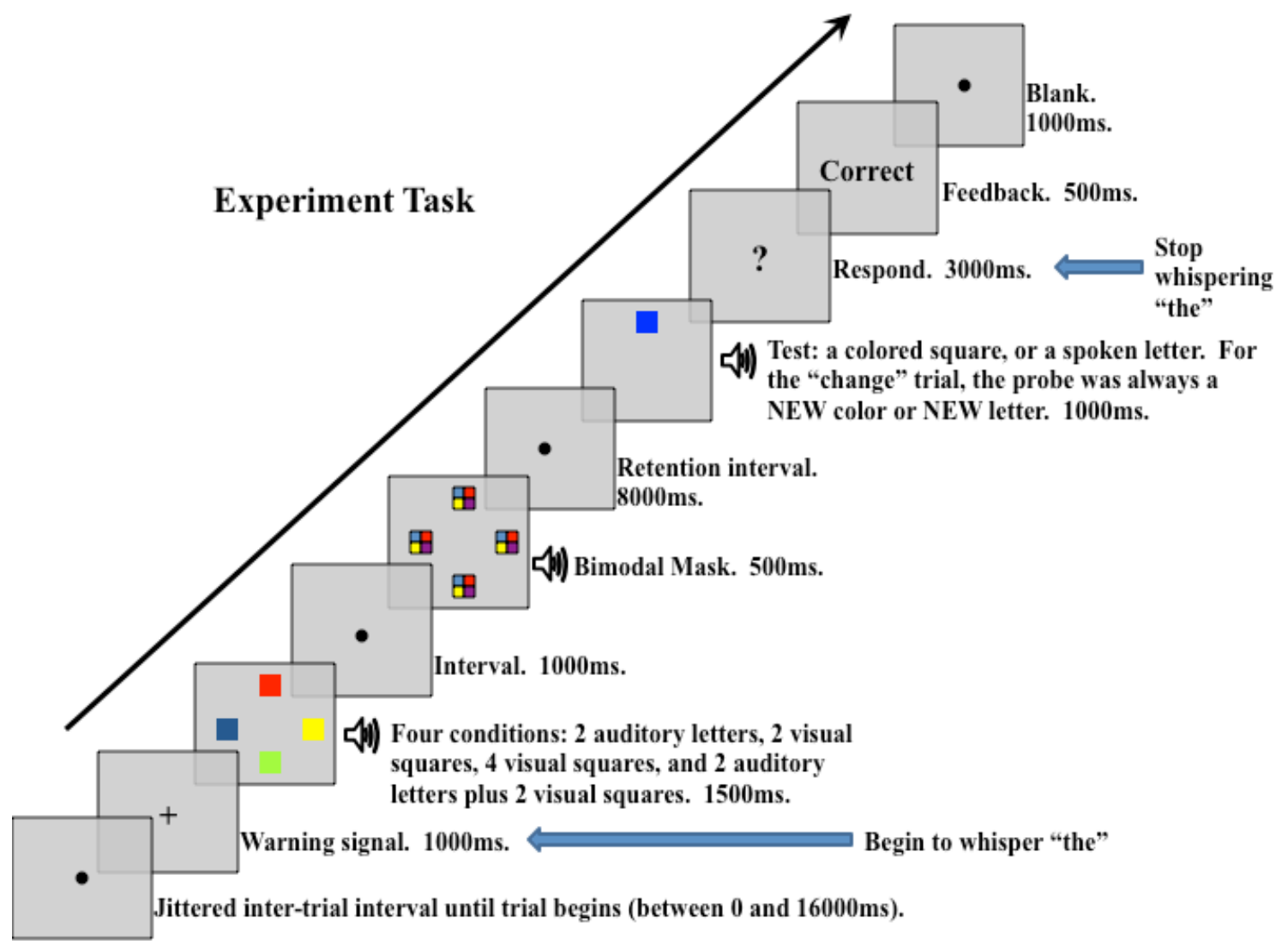

Figure 1. Experimental procedure of Experiment 2 in Cowan et al. (2011). 
Behavioral Procedure. Figure 1 shows the procedure of the experiment. The participants were instructed to perform a change-detection task, in which they remembered a few visual and/or auditory items for several seconds. Each trial started with a $1000 \mathrm{~ms}$ fixation, after which the participants were presented two auditory letters (2A), two visual colored squares (2V), two visual colored squares plus two auditory letters (2V2A), or four colored squares $(4 \mathrm{~V})$, for $1500 \mathrm{~ms}$. The two auditory letters were presented sequentially. Each auditory letter lasted $500 \mathrm{~ms}$, and there was a $250 \mathrm{~ms}$ interval between the two letters. The colored squares were presented simultaneously on the screen for $1500 \mathrm{~ms}$. Five hundred milliseconds visual and acoustic masks were simultaneously presented $1000 \mathrm{~ms}$ after the end of the stimulus presentation to eliminate traces of sensory memory. After a $8000 \mathrm{~ms}$ delay, a single test item was presented, and the participants were instructed to press a button to indicate whether this test item was the same as the remembered item at the same spatial location or verbal serial position as the test item, or was different from any item that they remembered. The test stimulus was presented for $1000 \mathrm{~ms}$, after which a "?" appeared on the screen. The participants had $3000 \mathrm{~ms}$ to respond to the test, after which feedback was provided. Additionally, to discourage verbal rehearsal, the participants were required to keep whispering the word "the" twice a second from the trial onset until the test stimulus appeared. Each trial lasted 18 seconds. Each participant performed 10 functional runs, and each run consisted of 16 trials (4 trials for each condition).

Neuroimaging Data Acquisition. The neuroimaging data was acquired with a $3 \mathrm{~T}$ Siemens Trio scanner at the Brain Imaging Center in the University of Missouri. For 
each participant, a T1-weighted and a T2-weighted high-resolution structural image were collected at the beginning of the scan session. Ten functional runs were then collected with a $\mathrm{T} 2 *$-weighted echo planar pulse sequence $(\mathrm{TR}=2000 \mathrm{~ms}, \mathrm{TE}=30 \mathrm{~ms}$, in-plane resolution $=4 \times 4 \mathrm{~mm}^{2}, 32$ axial slices with $4 \mathrm{~mm}$ thickness). Each functional run lasted 195 TRs.

Neuroimaging Data Preprocessing. The acquired neuroimaging data was preprocessed using AFNI (Cox, 1996). The preprocessing steps were: (1) removal of the first 2 volumes in each run, (2) time shifting the data to correct the deviations of slice acquisition time in each volume, (3) aligning all volumes to the first volume in the experiment, (4) removal of the signal spikes, (5) removal of mean, linear and quadratic trends in each run, and (6) normalizing all images into standard Talairach space. No spatial smoothing was performed.

Regions of Interest (ROIs) Definition. The left and right anterior IPS was selected as ROIs. The left anterior IPS ROI was selected as the left anterior IPS region (Talairach coordinates $-27,-46,31)$ reported in Figure 3 of Cowan et al. (2011), and the right anterior IPS ROI was selected as a counterpart of the left anterior IPS ROI in the right hemisphere. 
MVPA. MVPA was performed using the Princeton MVPA toolbox (code.google.com/p/ princeton-mvpa-toolbox), the Netlab open source toolbox (Bishop \& Nabney, 1996), and custom codes in MATLAB (MathWorks). Pairwise classification was performed, and the $2 \mathrm{~V}$ versus $2 \mathrm{~A}$ pair were used for the main analysis. The data were shifted by two time points ( 4 seconds) to accommodate for the hemodynamic delay. FMRI data corresponding to the WM maintenance period (time point 5 in a trial after shifting the data) were used to train a pattern classifier for each participant.

A three-layer (one hidden layer) feedforward backpropagation algorithm provided by the Netlab toolbox was used to train the classifiers. To compensate for the nondeterministic characteristics of the backpropagation algorithm, 50 iterations were performed and the average results were reported. The classifiers were trained to associate the activation patterns in the ROIs with either visual or auditory WM storage.

A leave-one-out cross-validation method was used to test the classification accuracy. Each participant performed ten runs in this experiment. For each crossvalidation iteration, nine runs were fed into the training algorithm, and the remaining run was used for testing the performance of the classifier. Each run in turn was selected as the testing run, and ten cross-validation iterations were conducted for each participant.

In the test, data of each time point in the $2 \mathrm{~V}$ and $2 \mathrm{~A}$ trials (nine time points in each trial) was assessed by the classifier. A classification accuracy value, ranging from 0 to 1 , was then calculated for each time point. A value of 0.5 denotes chance level. Thus, the outcome of the MVPA was a time course of classification accuracy for each time point in the trials. The classification accuracy value reflects the extent to which the 
classifier accurately predicted the cognitive state of each time point. The significance of classification accuracy at each time point was tested using one-tailed, one-sample t-test against the chance performance of 0.5 .

Similar to the $2 \mathrm{~V}$ versus $2 \mathrm{~A}$ conditions, the $2 \mathrm{~V} 2 \mathrm{~A}$ and the $4 \mathrm{~V}$ conditions both had a memory load of four and invoked the same level of BOLD signal in the left anterior IPS (Cowan et al., 2011). To further test the activation patterns in the IPS, the 2V2A and the $4 \mathrm{~V}$ conditions were test against each other in a pairwise classification to complement the $2 \mathrm{~V}$ versus $2 \mathrm{~A}$ classification.

\section{Results}

The time courses of group-averaged classification performance are shown in Figure 2. For the $2 \mathrm{~V}$ versus $2 \mathrm{~A}$ classification, both left (blue curve) and right anterior IPS (red curve) failed to distinguish between these two conditions at any time point in a trial. The $2 \mathrm{~V} 2 \mathrm{~A}$ versus $4 \mathrm{~V}$ classification showed the same results and is thus not shown in the figure. These results indicate that the activation patterns in left and right anterior IPS do not carry domain-specific information. 


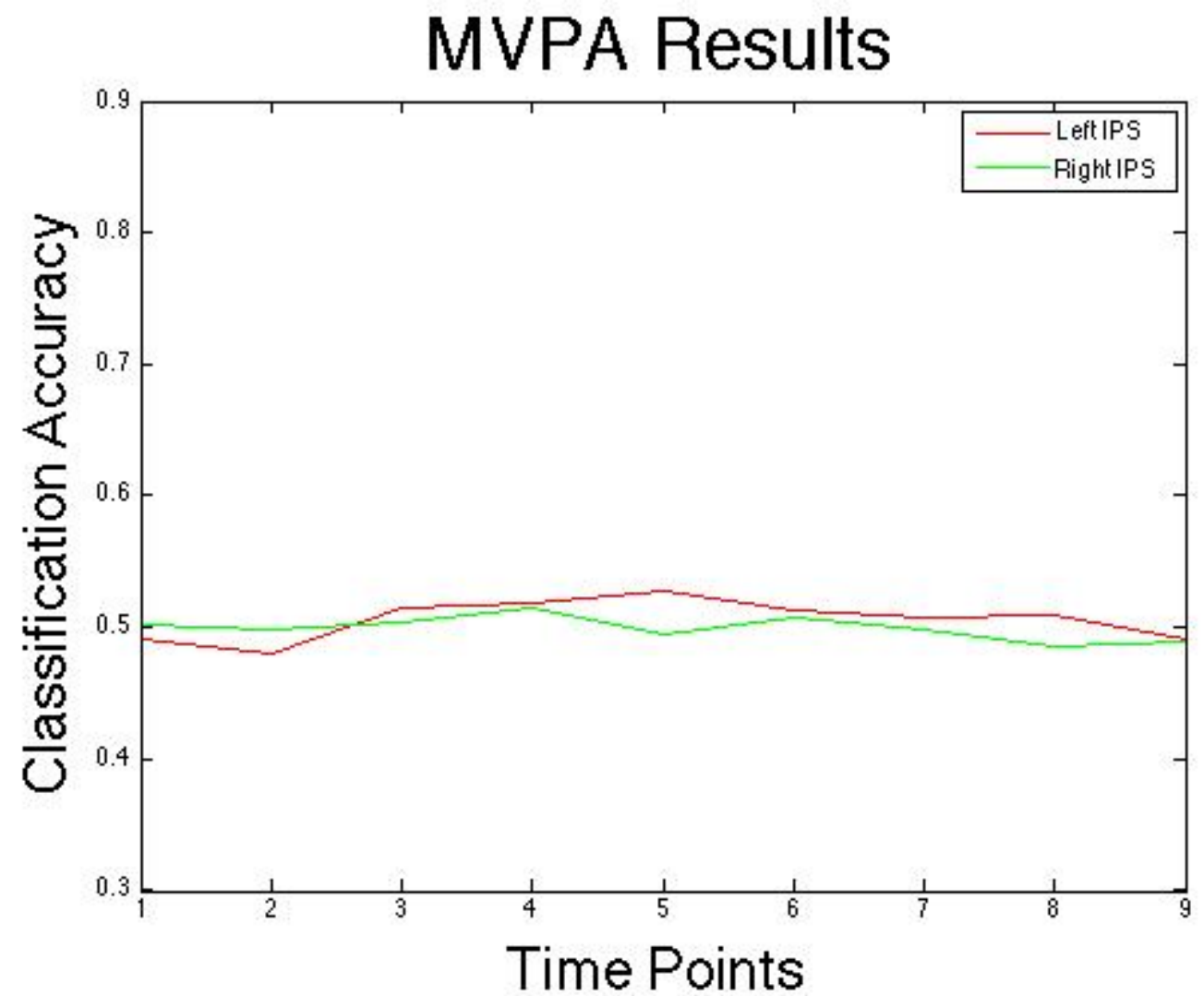

Figure 2. MVPA results. The $\mathrm{x}$ axis denotes the time points in a trial, and the $\mathrm{y}$ axis denotes the classification accuracy. Each curve denotes the dynamic classification accuracy in a certain ROI for a certain pairwise classification. Red: 2 visual versus 2 auditory, left anterior IPS ROI. Green: 2 visual versus 2 auditory, right anterior IPS ROI.

\section{Discussion}

Our previous research has shown that the overall activation level in a region in the left anterior IPS was sensitive to WM load and insensitive to stimulus domains, suggesting that this region is responsible for domain-general WM storage (Cowan et al., 2011). This result, however, does not exclude the possibility that the activation patterns in the left anterior IPS are different for visual and auditory WM. In this new analysis, MVPA was used to decode the activation patterns in the left and right anterior IPS, using the same 
data set as Experiment 2 in Cowan et al. (2011). The activation patterns in both left and right anterior IPS did not show any difference between the $2 \mathrm{~V}$ and $2 \mathrm{~A}$ conditions as well as between the $2 \mathrm{~V} 2 \mathrm{~A}$ and the $4 \mathrm{~V}$ condition. These results further support our previous finding that the left anterior IPS is responsible for domain-general WM storage (Cowan et al., 2011).

Some previous studies have used MVPA to decode the brain activation patterns in the IPS (Lewis-Peacock et al., 2012; Lewis-Peacock \& Postle, 2012; Riggall \& Postle, 2012). Riggall and Postle (2012) found that the IPS does not carry item-specific information in a visual WM task, which does not contradict our results. Our results, however, support a stronger claim that the left anterior IPS not only does not carry itemspecific information, but also does not carry domain-specific information.

Some previous MVPA studies found that the IPS codes domain information and is not a domain-general region. Tamber-Rosenau et al. (2013) analyzed several responseselection fMRI experiments in which the participants were instructed to perform a certain response when they were presented with a stimulus of a certain domain. Each domain (visual/auditory) was arbitrarily coupled with a vocal, manual, or oculomotor response. MVPA compared the activation patterns in several brain regions based on the stimulusresponse couplings in each experiment. The results showed that activation patterns in the IPS were able to distinguish between the stimulus-response domains, suggesting that this region may not code domain-general information. The discrepancy between TamberRosenau et al. (2013) and our study has two possible causes. (1) The response-selection task used in Tamber-Rosenau et al. (2013) involves not only WM but also arbitrary stimulus-response matching. The arbitrary coupling between a stimulus and a response 
could induce an abstract representation to the stimulus. The abstract representations, instead of the stimulus domains, might have caused the different activation patterns in the IPS. (2) Another possibility is that the IPS region in Tamber-Rosenau et al. (2013) is spatially lateral and posterior to the left anterior IPS used in our study. Given that different regions in the IPS are known to serve different processes (Culham \& Kanwisher, 2001), caution is advised when concluding the functions of the IPS based on the activation patterns in different sub-regions within the IPS during visual and auditory WM.

In sum, MVPA showed that the activation patterns in the left anterior IPS do not code domain-specific information, which lends further credence to our proposal that this region codes domain-general information in WM. This finding supports the domaingeneral account that stimuli of different domains share a common storage system in WM.

\section{PART II. DOMAIN-GENERAL AND DOMAIN-SPECIFIC NEURAL NETWORKS IN VISUAL AND AUDITORY WORKING MEMORY}

In Part I, we showed that a region in the left anterior IPS does not contain domainspecific information, suggesting that it is part of a domain-general storage system for both visual and auditory WM. It is unclear, however, how the other brain regions behave and interact with the IPS in the context of domain-specific and domain-general WM storage. An examination of neural networks is necessary to address this issue. Such examination, however, is overlooked in the previous literature. 
The majority of the previous WM studies have relied on univariate statistical approaches. Univariate approaches, such as the general linear model, are focused on the time series of each voxel independently and disregard the correlation between voxels. It can be argued, however, that the correlation between spatially distant brain voxels is a critical feature of fMRI datasets and reflects important information about the neural networks underlying cognitive tasks, which would not otherwise be evident using a univariate approach alone. Multivariate approaches take into account such inter-voxel correlations and thus may provide a more comprehensive view of the neural networks underlying WM.

An exploratory multivariate approach, constrained principal component analysis (CPCA), is used in this study to investigate the domain-general and domain-specific neural networks in WM. CPCA combines principal component analysis (PCA) with multivariate regression and has been used in neuroimaging studies on WM (Metzak et al., 2011, 2012; Woodward et al., 2006, 2013; for a complete introduction of the theory and applications of CPCA, see Hunter \& Takane, 2002; Takane \& Hunter, 2001). CPCA has several advantages over the other multivariate approaches. First, it is an exploratory whole-brain analysis approach and unlike some other approaches such as dynamic causal modeling and structural equation modeling, does not require a priori extraction of a certain groups of regions of interest. Second, unlike some other exploratory approaches such as PCA and independent component analysis, CPCA excludes the task-irrelevant variance and operates on the task-related variance in a data set. Finally, a finite impulse response (FIR)-based CPCA (Metzak et al., 2012), which is used in this analysis, is capable of tracking the dynamics of functional network over the entire trial. This is 
especially important in WM studies, in which the successive encoding and maintenance periods appear to recruit distinct functional networks (Woodward et al., 2006).

Application of multivariate techniques such as CPCA to fMRI data has already yielded valuable insights into other WM-related questions. For example, Woodward and colleagues conducted an fMRI study in which the participants remembered 2, 4, 6, or 8 letters for a delay of 3, 4, or 5 seconds (Woodward et al., 2006). Using CPCA, they found separate load-dependent functional networks for WM encoding and maintenance as well as strong negative correlation between the encoding and maintenance networks, which indicates complementary processes underlying WM encoding and maintenance. Importantly, past multivariate fMRI studies of WM (e.g., Abe et al., 2007; Chang et al., 2007; Cohen et al., 2012; Edin et al., 2007; Edin \& Klingberg, 2009; Fiebach et al., 2006; Gazzaley et al., 2007; Gazzaley et al., 2004; Habeck et al., 2012; Hampson et al., 2006, 2010; Honey et al., 2002; Kim et al., 2012; Kondo et al., 2004a, 2004b; Kuo et al., 2011; Lenartowicz \& McIntosh, 2005; Ma et al., 2012; Mayer et al., 2009; Palva et al., 2010; Payne \& Kounios, 2009; Rissman et al., 2008; Schlösser et al., 2006; Sundermann \& Pfleiderer, 2012) have used stimuli from only one sensory domain. Consequently, their potential value for answering the question of a domain-general versus domain-specific WM storage system is extremely limited.

In the present study, we bring multivariate techniques to bear on the question of whether the WM storage system is better conceptualized as a domain-specific or domaingeneral mechanism. Specifically, we applied CPCA to an existing fMRI dataset (Experiment 2 in Cowan et al., 2011) in which participants performed a task requiring them to remember stimuli from a single domain (i.e., only visual or only auditory) or 
multiple domains (both visual and auditory). The domain-specific theory would predict that, when both visual and auditory stimuli are used in the task, the WM storage system would recruit multiple neural networks, each of which includes specific brain areas for visual or auditory processing and shows distinct activity patterns for visual and auditory WM. In contrast, the domain-general theory would predict that a single neural network would be recruited for visual and verbal WM, with or without additional visual or verbal areas.

\section{Method}

For this study, we re-analyzed the data from Experiment 2 of Cowan et al. (2011), and a detailed description of the experiment can be found in that article. A brief summary of participants, experimental procedure (Figure 1) and neuroimaging methods can be found in the Method section of Part I in this dissertation.

Neuroimaging Data Preprocessing. The fMRI data were preprocessed using SPM8 (http://www.fil.ion.ucl.ac.uk/spm/software/spm8/). The preprocessing included slice-timing correction, head motion correction, co-registration of functional and anatomical images, spatial normalization into the Montreal Neurological Institute (MNI) space, resampling to isotropic 2-mm voxels, and spatial smoothing using a 6mm FWHM Gaussian filter. 
Neural Network Analysis. The univariate analysis method and results have been reported in Cowan et al. (2011). Below we describe the neural network analysis method.

The preprocessed data were analyzed using CPCA, which combines principal component analysis (PCA) with multivariate regression analysis. As a first (and critical) step, CPCA performs a multivariate regression on the entire fMRI dataset in order to extract a relatively pure estimate of task-related variance. The goal of this process is to remove nuisance variance related to task-irrelevant factors such as head movement and MR signal drift over time. In the multivariate regression, two matrices are generated: a data matrix $\mathrm{Z}$ and a design matrix $\mathrm{G}$. The data matrix $\mathrm{Z}$ contains the standardized BOLD signal, with each row representing one participant-specific volume and each column representing a voxel. The design matrix $\mathrm{G}$ contains timing of the experimental task conditions, with each row representing one participant-specific volume and each column representing one participant- and condition-specific predictor. With respect to the current analysis, the resulting matrix $\mathrm{Z}$ had 30880 rows and 189368 columns, and the matrix $\mathrm{G}$ had 30880 rows and 1024 columns. A finite impulse response (FIR) model was used to estimate the BOLD signal change over time. BOLD response for each condition and each participant was modeled by 16 predictors that cover a time duration of 32 seconds, with the first predictor corresponding to the trial onset. For each column of the G matrix, a value of 1 was assigned to the rows that are to be estimated by this predictor, and a value of 0 was assigned to the remaining rows.

Multivariate least-square linear regression is performed in which the data matrix $\mathrm{Z}$ is regressed on the design matrix $\mathrm{G}$ : 
$Z=G C+E$,

in which $\mathrm{C}$ contains each voxel's predictor weights for each experimental task condition, and $\mathrm{E}$ is the error matrix. The product of GC reflects the "true" variance relevant to the experimental design.

The second step of CPCA is to perform PCA on the GC matrix. This procedure identifies a set of orthogonal variables called principal components that explain a relatively large amount of variance in the data set. The number of selected principal components is usually less than the number of the original variables, and thus the dimensionality of the data set is reduced. The procedure involves generalized singular value decomposition of GC:

$U D V^{\prime}=G C$,

in which $\mathrm{D}$ is a diagonal matrix with nonnegative real numbers, known as singular values, on the diagonal, $\mathrm{U}$ is a matrix containing the left-singular vectors, and $\mathrm{V}$ is a matrix containing the right-singular vectors. Each column of the $\mathrm{V}$ matrix represents a functional network and could be mapped on a brain template to show the involved brain regions.

Condition-specific predictor weights are calculated in matrix $P(U=G P)$. The predictor weights in the matrix P represent the contribution of each predictor to each principal components for each participant, and thus allow for statistical tests of the effects of WM load condition and time points on the functional networks represented by the principal components. 
A more detailed illustration of the CPCA as applied in the current data set is provided in the Appendix.

\section{Results}

Four primary principal components were selected based on visual examination of the scree plot. The four components each accounted for $41.69 \%, 11.17 \%, 4.90 \%$, and $4.19 \%$, respectively, of the task-related variance. We believe that these components reflect, respectively, (1) domain-general encoding; (2) articulatory suppression, which was used in all conditions; (3) domain-general maintenance of information in WM; and (4) domain-specific visual encoding. Another important component, reflecting domainspecific auditory encoding, showed up sixth in the analysis and will also be described.

The functional networks represented by the two domain-general components, Components 1 and 3, are shown in Figure 3A and C, respectively. The predictor weights of Components 1 and 3, which depict the contribution of each component to the task conditions over time, are plotted in Figure $3 \mathrm{~B}$ and $\mathrm{D}$, respectively. The functional network represented by Component 2 is shown in Figure 4A, and its predictor weights are plotted in Figure 4B. The functional networks represented by the two domainspecific components, Components 4 and 6, are shown in Figure 5A and C, respectively. The predictor weights of Components 4 and 6 are plotted in Figure 5B and D, respectively. Figure $6 \mathrm{~A}, \mathrm{~B}, \mathrm{C}$, and D shows results of the post hoc Newman-Keuls tests comparing the predictor weights of each two memory conditions for components $1,3,4$, and 6 , respectively. 
Tables 1, 2, 3, and 4 contain the coordinates, Brodmann area, and size $\left(\mathrm{mm}^{3}\right)$ of each brain region for Components 1, 3, 4, and 6, respectively. (As detailed below, Component 2 appears to have limited theoretical significance and therefore was excluded from the aforementioned tables.)

Component 1: Domain-General Encoding. The Figure 3A shows the functional network represented by Component 1 . The functional network associated with component 1 consisted of several brain regions including bilateral lateral occipital cortex, left lingual gyrus, bilateral superior IPS, bilateral precuneus, bilateral superior temporal gyrus, right fusiform gyrus, left precentral gyrus, right premotor cortex, left dorsolateral prefrontal cortex, bilateral inferior frontal gyrus, bilateral medial frontal gyrus, bilateral anterior cingulate cortex, bilateral insula cortex, bilateral thalamus, and bilateral dorsal striatum. All brain regions showed positive loading values. 
Table 1. Brain regions included in Component 1 in the CPCA analysis (Part II).

\begin{tabular}{|c|c|c|c|c|c|}
\hline \multirow[t]{2}{*}{ Brain Region } & \multirow{2}{*}{$\begin{array}{l}\text { Brodmann's } \\
\text { Area }\end{array}$} & \multicolumn{3}{|c|}{$\begin{array}{l}\text { Peak MNI } \\
\text { coordinates }\end{array}$} & \multirow{2}{*}{$\begin{array}{l}\text { Volume } \\
\left(\mathrm{mm}^{3}\right)\end{array}$} \\
\hline & & $x$ & $y$ & $z$ & \\
\hline \multicolumn{6}{|l|}{ Positive component loadings } \\
\hline $\begin{array}{l}\text { Cluster 1: bilateral } \\
\text { Anterior Cingulate Cortex \& Medial } \\
\text { Frontal Gyrus }\end{array}$ & 6,32 & $\begin{array}{l}2 \\
0 \\
2\end{array}$ & $\begin{array}{l}18 \\
8 \\
-4\end{array}$ & $\begin{array}{l}60 \\
52 \\
68\end{array}$ & 29512 \\
\hline $\begin{array}{l}\text { Cluster 2: left hemisphere } \\
\text { Anterior Insula } \\
\text { Thalamus } \\
\text { Dorsal Striatum } \\
\text { Inferior Frontal Gyrus }\end{array}$ & $\begin{array}{l}13 \\
\text { N/A } \\
\text { N/A } \\
47\end{array}$ & $\begin{array}{l}-26 \\
-10 \\
-18 \\
-50\end{array}$ & $\begin{array}{l}14 \\
-18 \\
10 \\
20\end{array}$ & $\begin{array}{l}-6 \\
8 \\
2 \\
-12 \\
\end{array}$ & 11608 \\
\hline $\begin{array}{l}\text { Cluster 3: right hemisphere } \\
\text { Dorsal Striatum } \\
\text { Thalamus } \\
\text { Anterior Insula } \\
\end{array}$ & $\begin{array}{l}\mathrm{N} / \mathrm{A} \\
\mathrm{N} / \mathrm{A} \\
13\end{array}$ & $\begin{array}{l}16 \\
10 \\
24 \\
\end{array}$ & $\begin{array}{l}6 \\
-14 \\
18\end{array}$ & $\begin{array}{l}6 \\
8 \\
-6 \\
\end{array}$ & 8184 \\
\hline $\begin{array}{l}\text { Cluster 4: bilateral } \\
\text { Precuneus }\end{array}$ & 7 & 2 & -36 & 60 & 5952 \\
\hline $\begin{array}{l}\text { Cluster 5: right hemisphere } \\
\text { Premotor Cortex }\end{array}$ & 6 & 36 & -2 & 60 & 3904 \\
\hline $\begin{array}{l}\text { Cluster 6: left hemisphere } \\
\text { Lingual Gyrus }\end{array}$ & 18 & -2 & -72 & 6 & 3536 \\
\hline $\begin{array}{l}\text { Cluster 7: left hemisphere } \\
\text { Dorsolateral Prefrontal Cortex }\end{array}$ & 9 & -36 & 36 & 28 & 3200 \\
\hline $\begin{array}{l}\text { Cluster 8: right hemisphere } \\
\text { Fusiform Gyrus } \\
\text { Occipital Cortex }\end{array}$ & $\begin{array}{l}37 \\
19\end{array}$ & $\begin{array}{l}44 \\
42 \\
\end{array}$ & $\begin{array}{l}-48 \\
-74\end{array}$ & $\begin{array}{l}-24 \\
-12 \\
\end{array}$ & 3104 \\
\hline $\begin{array}{l}\text { Cluster 9: left hemisphere } \\
\text { Precentral Gyrus }\end{array}$ & 4 & $\begin{array}{l}-40 \\
-44\end{array}$ & $\begin{array}{l}-6 \\
-14\end{array}$ & $\begin{array}{l}56 \\
56\end{array}$ & 1720 \\
\hline $\begin{array}{l}\text { Cluster 10: right hemisphere } \\
\text { INTRAPARIETAL SULCUS }\end{array}$ & 7 & 32 & -54 & 62 & 1520 \\
\hline $\begin{array}{l}\text { Cluster 11: right hemisphere } \\
\text { Superior Temporal Gyrus }\end{array}$ & 22 & 48 & -32 & -4 & 960 \\
\hline $\begin{array}{l}\text { Cluster 12: right hemisphere } \\
\text { Inferior Frontal Gyrus } \\
\text { Superior Temporal Gyrus }\end{array}$ & $\begin{array}{l}47 \\
22 \\
\end{array}$ & $\begin{array}{l}50 \\
60\end{array}$ & $\begin{array}{l}22 \\
10\end{array}$ & $\begin{array}{l}-12 \\
-8\end{array}$ & 944 \\
\hline
\end{tabular}


Table 1 (continued)

\begin{tabular}{|l|l|l|l|l|l|}
\hline Brain Region & \multicolumn{2}{|l|}{$\begin{array}{l}\text { Brodmann's } \\
\text { Area }\end{array}$} & \multicolumn{2}{l|}{$\begin{array}{l}\text { Peak MNI } \\
\text { coordinates }\end{array}$} & \multicolumn{2}{l|}{$\begin{array}{l}\text { Volume } \\
\left(\mathrm{mm}^{3}\right)\end{array}$} \\
\hline $\begin{array}{l}\text { Cluster 13: left hemisphere } \\
\text { Occipital Cortex }\end{array}$ & 19 & -36 & -80 & -12 & 512 \\
\hline $\begin{array}{l}\text { Cluster 14: right hemisphere } \\
\text { Superior Temporal Gyrus }\end{array}$ & 22 & 64 & -10 & -4 & 360 \\
\hline $\begin{array}{l}\text { Cluster 15: left hemisphere } \\
\text { INTRAPARIETAL SULCUS }\end{array}$ & 7 & -30 & -56 & 60 & 336 \\
\hline $\begin{array}{l}\text { Cluster 16: left hemisphere } \\
\text { Superior Temporal Gyrus }\end{array}$ & 22 & -56 & -50 & 12 & 184 \\
\hline $\begin{array}{l}\text { Cluster 17: left hemisphere } \\
\text { Superior Temporal Gyrus }\end{array}$ & 22 & -56 & 2 & -8 & 80 \\
\hline
\end{tabular}


Table 2. Brain regions included in Component 3 in the CPCA analysis (Part II).

\begin{tabular}{|c|c|c|c|c|c|}
\hline \multirow[t]{2}{*}{ Brain Region } & \multirow{2}{*}{$\begin{array}{l}\text { Brodmann's } \\
\text { Area }\end{array}$} & \multicolumn{3}{|c|}{$\begin{array}{l}\text { Peak MNI } \\
\text { coordinates }\end{array}$} & \multirow[t]{2}{*}{$\begin{array}{l}\text { Volume } \\
\left(\mathrm{mm}^{3}\right)\end{array}$} \\
\hline & & $\mathrm{x}$ & $\mathrm{y}$ & $\mathrm{Z}$ & \\
\hline \multicolumn{6}{|l|}{ Positive component loadings } \\
\hline $\begin{array}{l}\text { Cluster 1: right hemisphere } \\
\text { Precentral Gyrus }\end{array}$ & 4 & 55 & 2 & 26 & 200 \\
\hline $\begin{array}{l}\text { Cluster 2: left hemisphere } \\
\text { INTRAPARIETAL SULCUS }\end{array}$ & 7 & -42 & -40 & 42 & 200 \\
\hline $\begin{array}{l}\text { Cluster 3: left hemisphere } \\
\text { Precentral Gyrus }\end{array}$ & 4 & -52 & -4 & 44 & 192 \\
\hline \multicolumn{6}{|l|}{ Negative component loadings } \\
\hline $\begin{array}{l}\text { Cluster 1: bilateral } \\
\text { Cuneus } \\
\text { Posterior Cingulate Cortex } \\
\text { Posterior Cingulate Cortex } \\
\text { Cuneus } \\
\text { Cuneus } \\
\text { Lingual Gyrus } \\
\text { Posterior Cingulate Cortex } \\
\text { Cuneus } \\
\text { Lingual Gyrus } \\
\text { Lingual Gyrus } \\
\text { Posterior Cingulate Cortex }\end{array}$ & $\begin{array}{l}18 \\
31 \\
31 \\
18 \\
18 \\
18 \\
31 \\
18 \\
18 \\
18 \\
31 \\
\end{array}$ & $\begin{array}{l}8 \\
-6 \\
4 \\
-2 \\
-10 \\
-10 \\
-4 \\
22 \\
10 \\
4 \\
-2 \\
\end{array}$ & $\begin{array}{l}-98 \\
-56 \\
-56 \\
-94 \\
-96 \\
-88 \\
-46 \\
-92 \\
-82 \\
-72 \\
-38 \\
\end{array}$ & $\begin{array}{l}20 \\
12 \\
8 \\
28 \\
20 \\
-10 \\
36 \\
24 \\
-8 \\
-4 \\
46 \\
\end{array}$ & 44000 \\
\hline $\begin{array}{l}\text { Cluster 2: bilateral } \\
\text { Ventral Medial Prefrontal Cortex } \\
\text { Anterior Prefrontal Cortex } \\
\text { Ventral Medial Prefrontal Cortex } \\
\text { Superior Frontal Gyrus } \\
\text { Ventral Medial Prefrontal Cortex }\end{array}$ & $\begin{array}{l}32 \\
10 \\
32 \\
6\end{array}$ & $\begin{array}{l}2 \\
-24 \\
-8 \\
-16 \\
-2\end{array}$ & $\begin{array}{l}60 \\
36 \\
62 \\
54 \\
46\end{array}$ & $\begin{array}{l}20 \\
44 \\
18 \\
34 \\
-6\end{array}$ & 25032 \\
\hline $\begin{array}{l}\text { Cluster 3: bilateral } \\
\text { Postcentral Gyrus } \\
\text { Postcentral Gyrus }\end{array}$ & $\begin{array}{l}1,2,3 \\
1,2,3\end{array}$ & $\begin{array}{l}-4 \\
2\end{array}$ & $\begin{array}{l}-48 \\
-28\end{array}$ & $\begin{array}{l}70 \\
74\end{array}$ & 3648 \\
\hline $\begin{array}{l}\text { Cluster 4: left hemisphere } \\
\text { Angular Gyrus }\end{array}$ & 39 & -44 & -74 & 32 & 1400 \\
\hline $\begin{array}{c}\text { Cluster 5: right hemisphere } \\
\text { Superior Frontal Gyrus }\end{array}$ & 6 & 16 & 48 & 42 & 864 \\
\hline
\end{tabular}


Table 3. Brain regions included in Component 4 in the CPCA analysis (Part II).

\begin{tabular}{|c|c|c|c|c|c|}
\hline \multirow[t]{2}{*}{ Brain Region } & \multirow{2}{*}{$\begin{array}{l}\text { Brodmann's } \\
\text { Area }\end{array}$} & \multicolumn{3}{|c|}{$\begin{array}{l}\text { Peak MNI } \\
\text { coordinates }\end{array}$} & \multirow{2}{*}{$\begin{array}{l}\text { Volume } \\
\left(\mathrm{mm}^{3}\right)\end{array}$} \\
\hline & & $x$ & $y$ & $z$ & \\
\hline \multicolumn{6}{|l|}{ Positive component loadings } \\
\hline $\begin{array}{l}\text { Cluster 1: bilateral } \\
\text { Cerebellum } \\
\text { Cerebellum } \\
\text { Cerebellum } \\
\end{array}$ & $\begin{array}{l}\text { N/A } \\
\text { N/A } \\
\text { N/A }\end{array}$ & $\begin{array}{l}40 \\
6 \\
-40 \\
\end{array}$ & $\begin{array}{l}-70 \\
-82 \\
-70\end{array}$ & $\begin{array}{l}-26 \\
-22 \\
-28 \\
\end{array}$ & 36344 \\
\hline $\begin{array}{l}\text { Cluster 2: bilateral } \\
\text { Medial Frontal Lobe }\end{array}$ & N/A & 18 & 30 & 0 & 4456 \\
\hline $\begin{array}{l}\text { Cluster 3: left hemisphere } \\
\text { Lateral Occipital Complex }\end{array}$ & 18 & -30 & -86 & 4 & 3392 \\
\hline $\begin{array}{l}\text { Cluster 4: left hemisphere } \\
\text { INTRAPARIETAL SULCUS }\end{array}$ & 7 & -28 & -62 & 64 & 2848 \\
\hline $\begin{array}{l}\text { Cluster 5: left hemisphere } \\
\text { Precentral Gyrus }\end{array}$ & 4 & -48 & -8 & 56 & 448 \\
\hline $\begin{array}{l}\text { Cluster 6: right hemisphere } \\
\text { Precuneus }\end{array}$ & 7 & 12 & -74 & 58 & 288 \\
\hline $\begin{array}{l}\text { Cluster 7: left hemisphere } \\
\text { Precuneus }\end{array}$ & 7 & -8 & -72 & 60 & 200 \\
\hline $\begin{array}{l}\text { Cluster 8: right hemisphere } \\
\text { INTRAPARIETAL SULCUS }\end{array}$ & 7 & 28 & -62 & 56 & 160 \\
\hline $\begin{array}{l}\text { Cluster 9: left hemisphere } \\
\text { Dorsolateral Prefrontal Cortex }\end{array}$ & 6 & -28 & -2 & 70 & 104 \\
\hline \multicolumn{6}{|l|}{ Negative component loadings } \\
\hline $\begin{array}{l}\text { Cluster 1: right hemisphere } \\
\text { Insula } \\
\text { Supramarginal Gyrus }\end{array}$ & $\begin{array}{l}\mathrm{N} / \mathrm{A} \\
40\end{array}$ & $\begin{array}{l}38 \\
66 \\
\end{array}$ & $\begin{array}{l}-16 \\
-24 \\
\end{array}$ & $\begin{array}{l}16 \\
36 \\
\end{array}$ & 9328 \\
\hline $\begin{array}{l}\text { Cluster 2: bilateral } \\
\text { Ventral Anterior Cingulate Cortex }\end{array}$ & 32 & -6 & 44 & 20 & 8096 \\
\hline $\begin{array}{l}\text { Cluster 3: left hemisphere } \\
\text { Insula }\end{array}$ & N/A & -34 & -18 & 16 & 5192 \\
\hline $\begin{array}{l}\text { Cluster 4: right hemisphere } \\
\text { Inferior Frontal Gyrus }\end{array}$ & $44,45,47$ & 52 & 22 & 16 & 1464 \\
\hline $\begin{array}{l}\text { Cluster 5: left hemisphere } \\
\text { Parahippocampal Gyrus }\end{array}$ & 28 & -24 & -12 & -30 & 624 \\
\hline $\begin{array}{l}\text { Cluster 6: left hemisphere } \\
\text { Supramarginal Gyrus }\end{array}$ & 40 & -64 & -30 & 36 & 304 \\
\hline
\end{tabular}


Table 4. Brain regions included in Component 6 in the CPCA analysis (Part II).

\begin{tabular}{|c|c|c|c|c|c|}
\hline \multirow[t]{2}{*}{ Brain Region } & \multirow{2}{*}{$\begin{array}{l}\text { Brodmann's } \\
\text { Area }\end{array}$} & \multicolumn{3}{|c|}{$\begin{array}{l}\text { Peak MNI } \\
\text { coordinates }\end{array}$} & \multirow[t]{2}{*}{$\begin{array}{l}\text { Volume } \\
\left(\mathrm{mm}^{3}\right)\end{array}$} \\
\hline & & $\mathrm{x}$ & $\mathrm{y}$ & $\mathrm{z}$ & \\
\hline \multicolumn{6}{|l|}{ Positive component loadings } \\
\hline $\begin{array}{r}\text { Cluster 1: right hemisphere } \\
\text { Superior Temporal Gyrus } \\
\text { Superior Temporal Gyrus } \\
\text { Superior Temporal Gyrus }\end{array}$ & $\begin{array}{l}22 \\
22 \\
22\end{array}$ & $\begin{array}{l}60 \\
62 \\
64\end{array}$ & $\begin{array}{l}-16 \\
-24 \\
-28\end{array}$ & $\begin{array}{l}-2 \\
4 \\
6\end{array}$ & 30264 \\
\hline $\begin{array}{l}\text { Cluster 2: left hemisphere } \\
\text { Superior Temporal Gyrus } \\
\text { Superior Temporal Gyrus }\end{array}$ & $\begin{array}{l}22 \\
22 \\
\end{array}$ & $\begin{array}{l}-54 \\
-48 \\
\end{array}$ & $\begin{array}{l}-26 \\
-30 \\
\end{array}$ & $\begin{array}{l}2 \\
6 \\
\end{array}$ & 25752 \\
\hline $\begin{array}{c}\text { Cluster 3: left hemisphere } \\
\text { Inferior Frontal Gyrus }\end{array}$ & 45 & -50 & 34 & 2 & 520 \\
\hline $\begin{array}{l}\text { Cluster 4: right hemisphere } \\
\text { Inferior Frontal Gyrus }\end{array}$ & 45 & 52 & 28 & 2 & 400 \\
\hline \multicolumn{6}{|l|}{ Negative component loadings } \\
\hline $\begin{array}{l}\text { Cluster 1: left hemisphere } \\
\text { Central Sulcus }\end{array}$ & 4 & -38 & -22 & 42 & 13048 \\
\hline $\begin{array}{l}\text { Cluster 2: right hemisphere } \\
\text { Middle Frontal Lobe }\end{array}$ & 6 & 20 & -20 & 66 & 4520 \\
\hline $\begin{array}{l}\text { Cluster 3: left hemisphere } \\
\text { Fusiform Gyrus }\end{array}$ & 20 & -44 & -14 & -34 & 560 \\
\hline $\begin{array}{l}\text { Cluster 4: left hemisphere } \\
\text { Thalamus }\end{array}$ & $\mathrm{N} / \mathrm{A}$ & -24 & -16 & 12 & 208 \\
\hline $\begin{array}{l}\text { Cluster 5: left hemisphere } \\
\text { Parahippocampal Gyrus }\end{array}$ & 35 & -32 & -6 & -26 & 120 \\
\hline
\end{tabular}



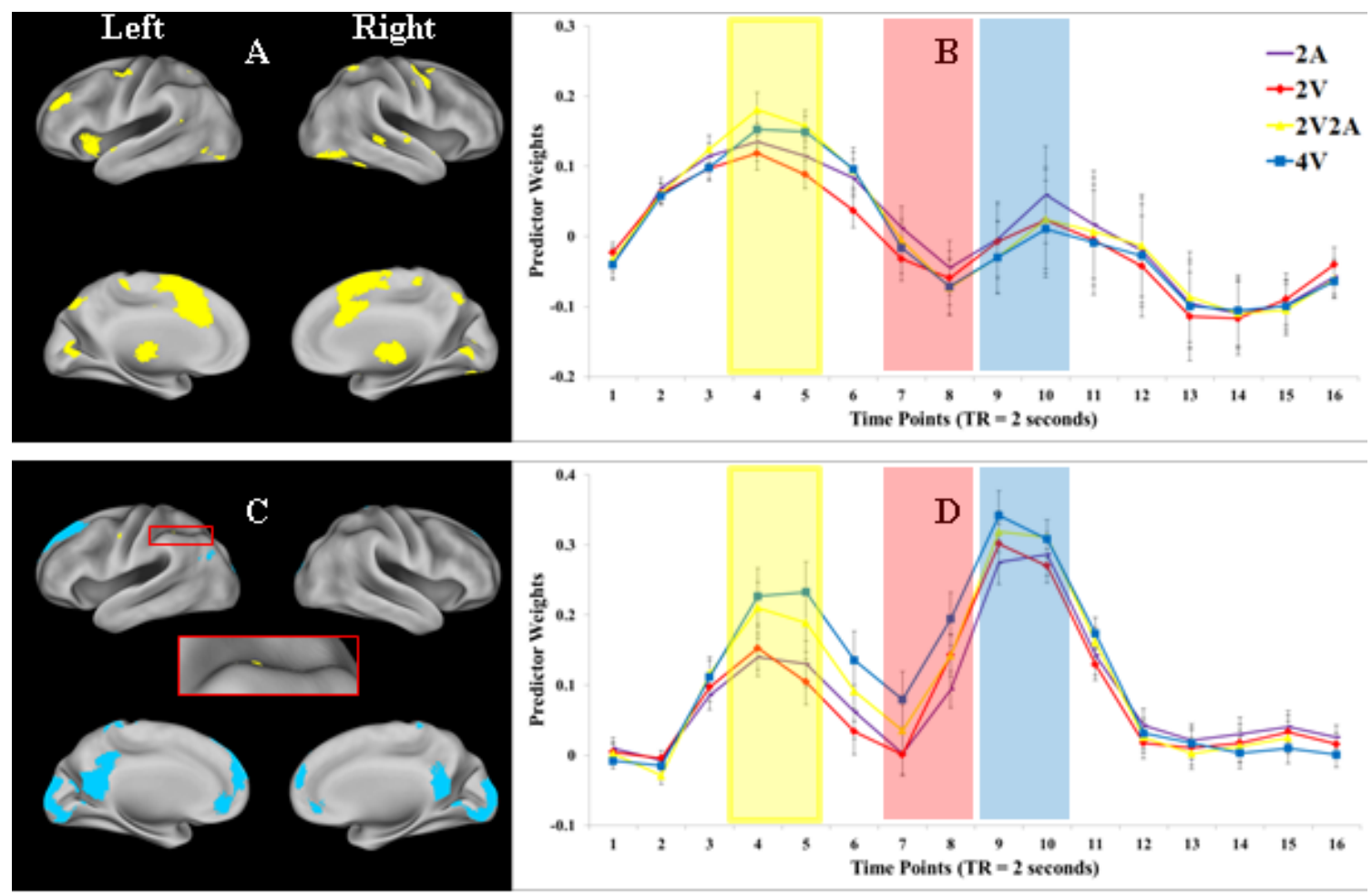

Figure 3. Brain networks and predictor weights of two domain-general components (Components 1 and 3). $\mathrm{A}$ and $\mathrm{C}$ : brain regions of Components 1 and 3, respectively. The region in the red square in $\mathrm{C}$ shows the left anterior IPS. Only voxels with the most extreme 5\% component loadings (whether positive or negative loadings) are displayed. Voxels with positive loadings are shown as yellow, and voxels with negative loadings are shown as blue. Results are visualized on an inflated PALS-B12 fiducial atlas (Van Essen, 2005; Van Essen \& Dierker, 2007) provided by the Caret software (http://www.nitrc.org/projects/caret/; Van Essen et al., 2001). B and D: mean predictor weights over time (TR $=2$ seconds) of Components 1 and 3, respectively. Red curve: 2 visual items (2V). Blue curve: 4 visual items $(4 \mathrm{~V})$. Yellow curve: 2 visual and 2 auditory items $(2 \mathrm{~V} 2 \mathrm{~A})$. The yellow, red, and blue rectangles denote encoding, maintenance, and response periods, respectively. The error bars represent standard errors.

Figure 3B shows the peri-stimulus predictor weights for each WM load condition.

Visual inspection of the plots showed that this network had the highest predictor weights during WM encoding, which seem to depend on the number of items to be encoded into WM regardless of sensory domain. A two-way repeated measure ANOVA with WM load conditions (2A, 2V, 2V2A, and 4V) and time points (1 to 16) as within-participant factors showed a significant main effect of time points, $F(15,225)=5.77, p<.001, \eta_{\mathrm{p}}=$ .28 , as well as a significant interaction of WM load condition by time points, $F(45,675)$ $=1.87, p<.001, \eta_{p}=.11$. The main effect of time points was mainly due to the increased 
predictor weights during encoding compared with those during maintenance and probe (Figure 3B).

Post hoc Newman-Keuls test of the interaction effect revealed that the $2 \mathrm{~A}$ and the $2 \mathrm{~V}$ conditions showed almost identical predictor weights across the trial, and that the 2V2A and the $4 \mathrm{~V}$ conditions also showed almost identical predictor weights across the trial (Figure 6A). Importantly, the interaction was caused by higher predictor weights for the $2 \mathrm{~V} 2 \mathrm{~A}$ condition versus the $2 \mathrm{~V}$ and the $2 \mathrm{~A}$ conditions, as well as higher predictor weights for the $4 \mathrm{~V}$ condition versus the $2 \mathrm{~V}$ condition during the encoding phase, suggesting that this brain network is sensitive to domain-general WM load during encoding. The $4 \mathrm{~V}$ versus $2 \mathrm{~A}$ contrast was not significant, though the means were in the anticipated order.

Component 2: Articulatory Suppression. Figure 4A shows the functional network represented by Component 2. Both positive and negative brain regions were included in this network. The positive brain regions included bilateral inferior frontal gyrus, bilateral middle frontal gyrus, right superior and middle temporal gyrus, bilateral caudate nucleus, and right inferior temporal gyrus. The negative brain regions included left postcentral gyrus, as well as areas around the brain and near the medial line. Importantly, there were no differences between conditions in this component (Figure 4B). This limits its theoretical significance; it is presented here only because it accounts for the second-highest amount of variance overall. It is likely to represent the brain activity related to articulatory suppression (whispering "the" repeatedly), which was carried out 
from the beginning of each trial through the manual response to prevent covert verbal rehearsal, but was not carried out between trials. There also were some indications of movement artifact from this suppression task (Birn et al., 2005; Yetkin et al., 1996) that had been subtracted out for univariate analyses reported by Cowan et al. (2011).
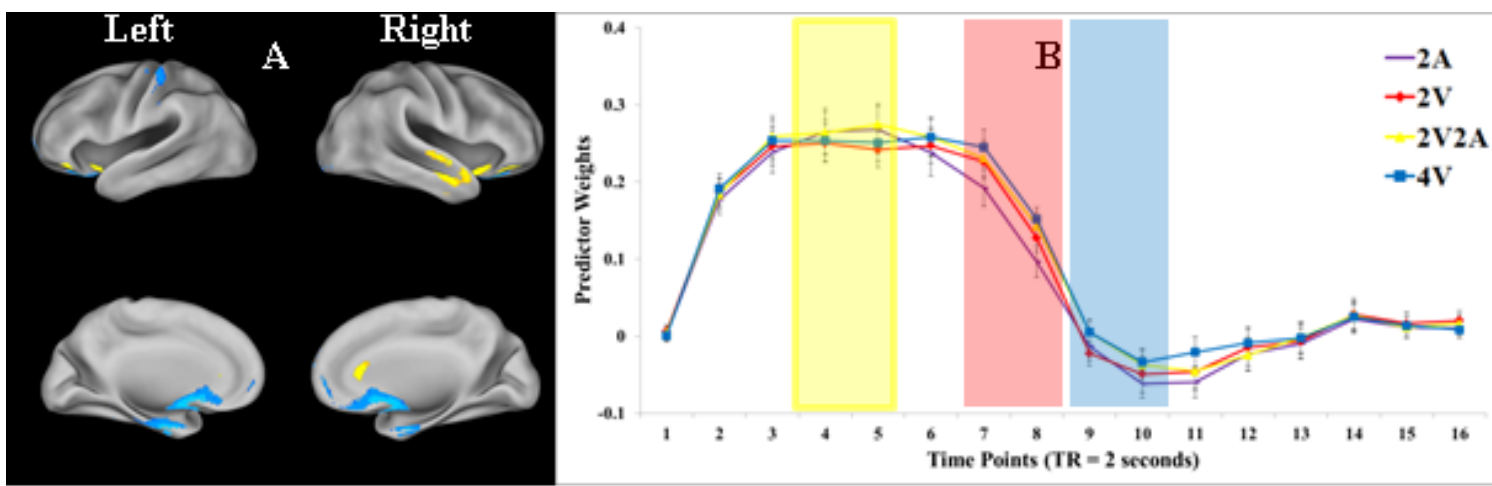

Figure 4. Brain networks and predictor weights of Component 2. A: brain regions of Component 2. Only voxels with the most extreme $5 \%$ component loadings (whether positive or negative loadings) are displayed. Voxels with positive loadings are shown as yellow, and voxels with negative loadings are shown as blue. Results are visualized on an inflated PALS-B12 fiducial atlas (Van Essen, 2005; Van Essen \& Dierker, 2007) provided by the Caret software (http://www.nitrc.org/projects/caret/; Van Essen et al., 2001). B: mean predictor weights over time ( $\mathrm{TR}=2$ seconds) of Component 2 . Red curve: 2 visual items $(2 \mathrm{~V})$. Blue curve: 4 visual items (4V). The yellow, red, and blue rectangles denote encoding, maintenance, and response periods, respectively. Yellow curve: 2 visual and 2 auditory items (2V2A). The error bars represent standard errors.

Component 3: Domain-General Maintenance. Figure 5C shows the functional network represented by Component 3. Both positive and negative brain regions were included in this network. The positive brain regions included left IPS and bilateral precentral gyrus. The negative brain regions included bilateral posterior cingulate cortex, left inferior parietal lobule, bilateral occipital cortex, bilateral postcentral gyrus, bilateral superior frontal gyrus, bilateral ventral cingulate cortex, and bilateral ventral medial prefrontal cortex. 
Visual inspection of the predictor weight plots in Figure 5D suggests that this network is sensitive to multimodal WM load during encoding, maintenance, and retrieval periods. A two-way repeated measure ANOVA with WM load conditions $(2 \mathrm{~A}, 2 \mathrm{~V}$, $2 \mathrm{~V} 2 \mathrm{~A}$, and $4 \mathrm{~V}$ ) and time points (1 to 16 ) as within-participant factors showed a significant main effects of WM load conditions, $F(3,45)=10.03, p<.001, \eta_{\mathrm{p}}=.40$, and time points, $F(15,225)=16.91, p<.001, \eta_{p}=.53$. As illustrated in Figure $6 \mathrm{~B}$, the main effect of WM load conditions was due to higher overall predictor weights for the $2 \mathrm{~V} 2 \mathrm{~A}$ and the $4 \mathrm{~V}$ conditions compared with those for the $2 \mathrm{~A}$ and the $2 \mathrm{~V}$ conditions, as well as higher overall predictor weights for the $4 \mathrm{~V}$ condition compared with those for the $2 \mathrm{~V} 2 \mathrm{~A}$ condition. The main effect of time points was due to an "M"-shaped time course in which the predictor weights were higher during encoding and probe and lower during maintenance (Figure 3D).

The interaction between WM load conditions and time points was also significant, $F(45,675)=5.79, p<.001, \mathrm{n}_{\mathrm{p}}=.28$. Post-hoc Newman-Keuls test revealed that the $2 \mathrm{~A}$ and the $2 \mathrm{~V}$ conditions showed almost identical predictor weights across the trial, whereas the $2 \mathrm{~V} 2 \mathrm{~A}$ and the $4 \mathrm{~V}$ conditions showed similar predictor weights across the trial except that the 2V2A condition exhibited slightly but significantly smaller predictor weights than the $4 \mathrm{~V}$ condition during the maintenance period. Importantly, both the $2 \mathrm{~V} 2 \mathrm{~A}$ and the $4 \mathrm{~V}$ conditions showed significantly higher predictor weights than the $2 \mathrm{~A}$ and the $2 \mathrm{~V}$ conditions at most time points representing WM encoding, maintenance, and retrieval (Figure 6B). Thus, brain regions in this neural network are sensitive to domain-general WM load during all stages of WM processing. 
Component 4: Domain-Specific Visual Encoding. Figure 5A illustrates the functional network represented by Component 4. Both positive and negative brain regions were included in this network. The positive brain regions included bilateral posterior IPS, bilateral precuneus, left occipital cortex, left precentral gyrus, left dorsolateral prefrontal cortex, bilateral medial frontal gyrus, and bilateral cerebellum. The negative brain regions included bilateral supramarginal gyrus, right inferior frontal gyrus, bilateral ventral anterior cingulate cortex, bilateral insula, and left parahippocampal gyrus.

Visual inspection of the predictor weights plots showed that this brain network is sensitive to visual load during WM encoding but not maintenance and retrieval (Figure 5B). A two-way repeated measure ANOVA with WM load conditions $(2 \mathrm{~A}, 2 \mathrm{~V}, 2 \mathrm{~V} 2 \mathrm{~A}$, and $4 \mathrm{~V}$ ) and time points (1 to 16) as within-participant factors showed a significant main effects of WM load conditions, $F(3,45)=8.07, p<.001, \eta_{p}=.35$, and time points, $F(15$, $225)=9.63, p<.001, \eta_{\mathrm{p}}=.39$. The main effect of WM load conditions was primarily due to higher overall predictor weight for the $4 \mathrm{~V}$ condition compared with those for the other three conditions (Figure 6C). The main effect of time points appears to be due to elevated predictor weights during WM encoding and maintenance, but not during retrieval (Figure 5B).

The interaction between WM load conditions and time points was also significant, $F(45,675)=14.73, p<.001, \eta p=.50$. Post hoc Newman-Keuls test showed that the interaction was mainly due to differences of predictor weights caused by visual load during the encoding period. During the encoding period represented by approximately time points 4 and 5 , the predictor weights for the $4 \mathrm{~V}$ condition were higher than those for 
the $2 \mathrm{~V} 2 \mathrm{~A}$ and the $2 \mathrm{~V}$ condition, which in turn were higher than those for the $2 \mathrm{~A}$

condition (Figure 6C). After encoding, the predictor weights for different WM load

conditions converged and showed smaller differences. Although the $4 \mathrm{~V}$ condition

continued to show higher predictor weights than the $2 \mathrm{~V}$ condition throughout the

maintenance window, this load effect did not generalize to the other contrasts, such as $4 \mathrm{~V}$

$-2 \mathrm{~V} 2 \mathrm{~A}, 2 \mathrm{~V} 2 \mathrm{~A}-2 \mathrm{~A}$, and $2 \mathrm{~V}-2 \mathrm{~A}$, during the maintenance period. It thus seems likely

that this neural network is responsible for processing visual information during WM

encoding.
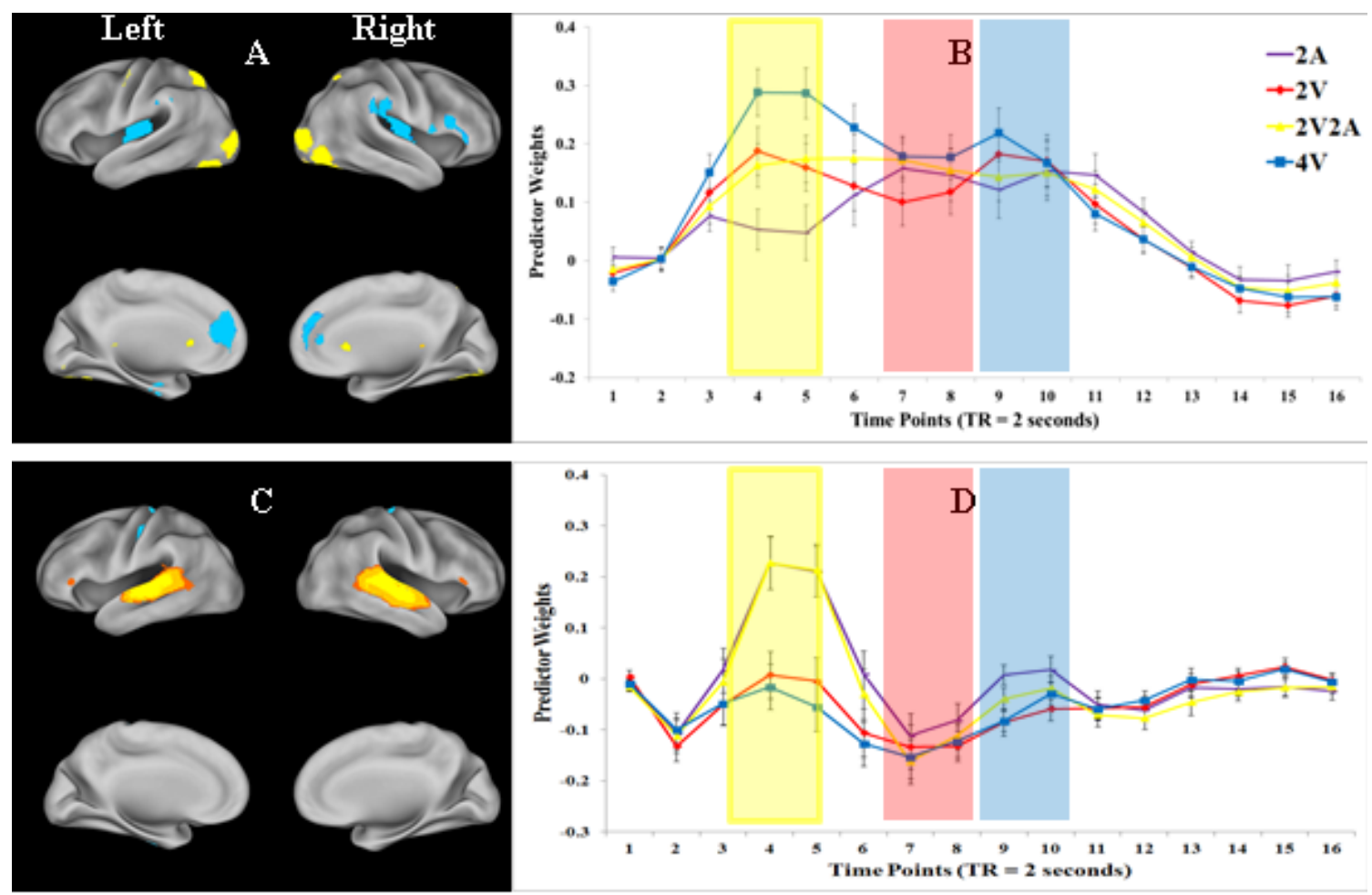

Figure 5. Brain networks and predictor weights of two domain-specific components (Components 4 and 6). $\mathrm{A}$ and $\mathrm{C}$ : brain regions of Components 4 and 6, respectively. Only voxels with the most extreme 5\% component loadings (whether positive or negative loadings) are displayed. Voxels with positive loadings are shown as yellow, and voxels with negative loadings are shown as blue. Results are visualized on an inflated PALS-B12 fiducial atlas (Van Essen, 2005; Van Essen \& Dierker, 2007) provided by the Caret software (http://www.nitrc.org/projects/caret/; Van Essen et al., 2001). B and D: mean predictor weights over time ( $T R=2$ seconds) of Components 4 and 6 , respectively. Red curve: 2 visual items (2V). Blue curve: 4 visual items $(4 \mathrm{~V})$. The yellow, red, and blue rectangles denote encoding, maintenance, and 
response periods, respectively. Yellow curve: 2 visual and 2 auditory items (2V2A). The error bars represent standard errors.

\section{Component 6, An Additional Component-of-Interest: Domain-Specific Auditory}

Encoding. In order to identify additional components that may have shown insightful patterns but did not explain a relatively large amount of variance, we extracted ten components in a separate analysis. Besides Components 1 to 4, only Component 6 showed WM load-dependent predictor weights. Therefore, another analysis was conducted in which six components were extracted. The result showed that Component 6 accounted for $2.3 \%$ of the task-related variance. Figure 5C shows the neural network represented by Component 6 . This neural network included both positive and negative brain regions. The positive brain regions included bilateral superior temporal gyrus and bilateral inferior frontal gyrus. The negative brain regions included bilateral precentral and postcentral gyrus, left fusiform gyrus, left thalamus, and left parahippocampal gyrus.

Figure 5D shows the predictor weights for each WM load condition in Component 6. Visual inspection of the plots shows that this network was sensitive to auditory load during only the encoding period. A two-way repeated measure ANOVA with WM load conditions ( $2 \mathrm{~A}, 2 \mathrm{~V}, 2 \mathrm{~V} 2 \mathrm{~A}$, and $4 \mathrm{~V})$ and time points (1 to 16$)$ as withinparticipant factors showed a significant main effects of WM load conditions, $F(3,45)=$ $17.91, p<.001, \eta_{\mathrm{p}}=.54$, and time points, $F(15,225)=7.66, p<.001, \eta_{\mathrm{p}}=.34$. The main effect of WM load conditions was due to higher overall predictor weights for the $2 \mathrm{~A}$ and the $2 \mathrm{~V} 2 \mathrm{~A}$ conditions compared with those for the $2 \mathrm{~V}$ and the $4 \mathrm{~V}$ conditions (Figure 6D). The main effect of time points was due to elevated average predictor weights during the encoding period (Figure 5D). 
The interaction between WM load conditions and time points was also significant, $F(45,675)=21.08, p<.001, \eta_{p}=.58$. Post hoc Newman-Keuls test showed that the interaction was primarily due to higher predictor weights for the $2 \mathrm{~A}$ and the $2 \mathrm{~V} 2 \mathrm{~A}$ conditions than those for the $2 \mathrm{~V}$ and the $4 \mathrm{~V}$ conditions during only the encoding period (Figure 6D). This neural network is therefore likely to be responsible for encoding auditory stimuli into WM. 


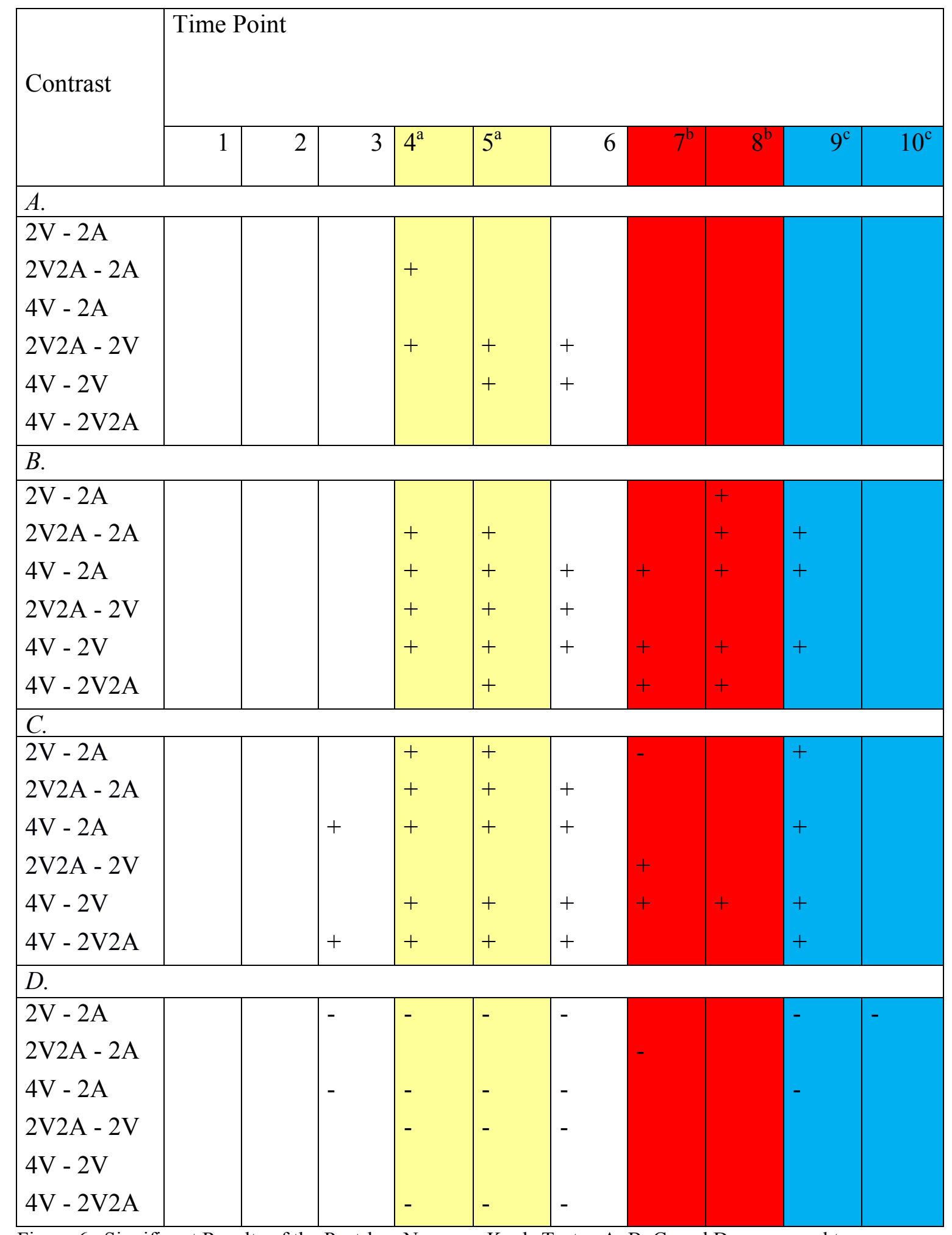

Figure 6. Significant Results of the Post-hoc Newman-Keuls Tests. A, B, C, and D correspond to components $1,3,4$, and 6 , respectively. The rows are pairwise contrasts between WM load conditions. The columns are 10 time points, in which time point 1 refers to the onset of a trial. The "+" sign denotes a 
significant positive contrast, and the "-"sign denotes a significant negative contrast. 2V: 2 visual items. 2A: 2 auditory items. 4V: 4 visual items. 2V2A: 2 visual plus 2 auditory items. Time points 11-16 are not included in this figure, because these time points correspond mainly to the undershoot period of the hemodynamic function which does not carry a significant amount of task-related information. Very few contrasts were significant during this time period, the only ones being $2 \mathrm{~V}-2 \mathrm{~A}$ at time point 11 and $4 \mathrm{~V}-2 \mathrm{~A}$ at

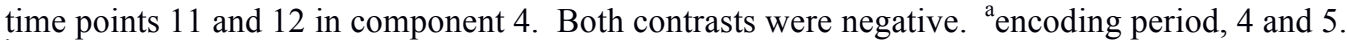
${ }^{b}$ maintenance periods, 7 and 8 . ' response periods, 9 and 10 .

\section{Discussion}

This study investigated the functional networks underlying a multimodal changedetection experiment. An exploratory multivariate data analysis method, CPCA, was used to extract neural networks underlying the WM task.

CPCA revealed distinct brain networks sensitive to domain-general versus domain-specific processes. On one hand, two components demonstrated characteristics consistent with domain-general load dependency: Component 1 was sensitive to both visual and auditory loads during only the encoding period, and Component 3 was sensitive to both visual and auditory loads throughout the trial. On the other hand, the results for two other components were consistent with domain-specific load dependency: Component 4 was sensitive to visual load (but not auditory load) during the encoding period, and Component 6 showed dependency on auditory load (but not visual load) during the encoding period. These findings are consistent with the assertion that different neural networks are involved in domain-general and domain-specific processes in the WM task.

The two domain-general components, Components 1 and 3, showed different patterns of predictor weights and consisted of different brain regions. Component 1 was sensitive to visual and auditory load during only the encoding period and included 
primary sensory regions as well as several association areas including the IPS, the precuneus, the dorsolateral prefrontal cortex, the anterior insula, the premotor cortex, and the dorsal anterior cingulate cortex. Most of these brain regions, such as the precuneus, the dorsolateral prefrontal cortex, the premotor cortex, and the dorsal anterior cingulate cortex, are frequently found to be active during WM encoding (Cairo et al., 2004; Habeck et al., 2005; Woodward et al., 2006). Therefore, Component 1 seems to represent a neural network for domain-general encoding.

In contrast, Component 3 was sensitive to visual and auditory load throughout the trial and consisted of both negative and positive regions. The negative brain regions overlapped with the default mode network which shows activation during rest and deactivation during attention-demanding tasks (Buckner et al., 2008). Given that the behavioral results have shown that the 4-item conditions were more demanding than the 2-item conditions regardless of modalities (Cowan et al., 2011), it is not surprising that the default mode network showed negative bimodal load-dependency throughout the entire trial. This network also consisted of several brain regions with positive loadings, among which was a subregion within the left anterior IPS (Figure 3C, expanded region). Critically, this left anterior IPS subregion (MNI coordinates: $-42,-40$, 42; Talairach coordinates: $-40,-42,37$, converted using icbm2tal, Lancaster et al., 2007) is spatially close to the left anterior IPS subregion (Talairach coordinates: -27, -46, 31) identified in the univariate analysis, which was the only brain region sensitive to bimodal load during the maintenance period (see Figure 3 in Cowan et al., 2011). This result from the CPCA analysis thus reinforces the results from the univariate analysis. Only a small region within the precentral gyrus was found to be positively connected with the left anterior IPS 
in this network, although there were several negative brain regions as noted. When we relaxed the criterion to examine the brain regions with top $10 \%$ loading values, however, we found that the positive brain regions also included bilateral dorsal anterior cingulate cortex, which has been suggested to modulate activities in the lateral frontal and the parietal regions (Bush et al., 2000). It thus seems that the left anterior IPS, the dorsal anterior cingulate cortex, and the precentral gyrus form a network responsible for domain-general WM maintenance.

Unlike the domain-general components, the two domain-specific components, Components 4 and 6 , showed similar patterns of predictor weights: both components were sensitive to domain-specific load during the encoding period. Component 4 was sensitive to visual load during encoding and included the lateral occipital cortex and the posterior IPS. The lateral occipital cortex is widely accepted to be responsible for visual object recognition (Grill-Spector et al., 2001). The posterior IPS is spatially distinct from the anterior IPS discovered in Component 3. The posterior IPS has been reported to be sensitive to visual object complexity (Xu \& Chun, 2006), to be dependent on visual load in a perceptual task with no memory requirements (Mitchell \& Cusack, 2008), and to be structurally connected to the superior occipital lobe (Uddin et al., 2010). Interestingly, a similar posterior IPS region was previously proposed to be responsible for visual WM maintenance (Todd \& Marois, 2004). Combined with our findings, we could suggest that this region carries visual- specific encoding information that under some circumstances can also contribute to visual working memory. An alternative possibility is that different subregions within the posterior IPS, which are indistinguishable with 
current experimental paradigms and techniques, are involved in WM encoding versus maintenance.

Component 6 was sensitive to auditory load and consisted of bilateral superior temporal gyrus and inferior frontal gyrus. The superior temporal gyrus is responsible for auditory processing (Demonet et al., 1992). The left inferior frontal gyrus is important for verbal comprehension and is frequently reported to be active during verbal WM encoding and maintenance (Cohen et al., 1997; Nixon et al., 2004). The latter region, however, was not necessarily recruited during verbal WM maintenance in this study, given that Component 6 was not sensitive to auditory load during the maintenance period.

It is thus clear that different neural networks were responsible for domain-general and domain-specific processes in WM. On one hand, the domain-specific networks showed load-dependent patterns during only the encoding period, suggesting their roles in domain-specific encoding. On the other hand, the domain-general network represented by Component 1 showed load-dependent patterns during the encoding period, whereas the domain-general network represented by Component 3 showed load-dependent patterns throughout the entire trial.

WM encoding recruited both domain-specific (Components 4 and 6) and domaingeneral networks (Component 1). These networks are likely to be involved in both perceptual encoding and WM consolidation. One possibility is that the domain-specific networks are involved in perceptual encoding, and that the domain-general network subsequently consolidates the perceptual representations into WM. This possibility is partly supported by the fact that the onset of the load effect was earlier in the domain- 
specific networks than in the domain-general network (Figure 6A, C, D). Elucidation of the exact functions of these networks, however, requires more investigation.

In contrast to WM encoding, WM maintenance recruited only a domain-general network, which supports the view that WM storage is a unitary system that maintains visual and auditory items through the same processes (Cowan, 1995; Saults \& Cowan, 2007). The fact that no domain-specific brain network was involved in WM maintenance, however, does not necessarily imply that WM storage does not recruit any stimulus-specific process. In fact, a few recent studies used multi-voxel pattern analysis to decode the BOLD signal and found that the posterior sensory regions showed stimulus-specific activity patterns during WM maintenance (Harrison \& Tong, 2009; Lewis-Peacock \& Postle, 2012; Riggall \& Postle, 2012). These studies proposed that the posterior sensory regions function in a sub-threshold manner which cannot typically be detected by traditional analysis methods. It is thus possible that the posterior sensory regions receive top-down regulations from the multimodal network, especially from the IPS, during WM maintenance. In this way, both lower-level stimulus-specific and higher-level categorical information are kept in WM.

Contrary to the previous finding that similar brain regions underlie WM encoding and maintenance (Pessoa et al., 2002), this study revealed different load-dependent domain-general networks during WM encoding and WM maintenance. This result supports the counter-viewpoint that WM consolidation during encoding is independent from WM maintenance (Woodman \& Vogel, 2005). 
Interestingly, several subregions within the IPS were functionally connected to different brain regions and showed different properties in this study. Component 3 included a small region within the left anterior IPS that worked with several frontal regions and showed domain-general WM load dependency during multiple stages in the WM tasks. Component 4 included another region within the bilateral posterior IPS that was functional connected to primarily the lateral occipital cortex and was sensitive to only visual load during the encoding periods. Component 1 included a region within the bilateral posterior IPS that was functionally connected to both posterior sensory regions and multiple frontal and subcortical regions and was sensitive to domain-general load during only the encoding period. Importantly, these three IPS subregions showed little overlap, consistent with the view that the IPS consists of multiple subregions each with distinct (Culham \& Kanwisher, 2001; Xu \& Chun, 2006). This finding is also supported by a resting-state and structural connectivity study by Uddin and colleagues (Uddin et al., 2010), which partitioned the IPS into three parts and found that the two anterior parts showed greater resting-state functional connectivity and structural connectivity with prefrontal regions, and that the posterior part showed greater resting-state functional connectivity and structural connectivity with extrastriate visual areas.

In summary, the present application of CPCA to data from a multi-modal working memory fMRI study revealed multiple functional networks. By using simultaneous visual and auditory stimuli in a single trial, this study allowed for evaluation of separate domain-general and domain-specific networks underlying different processes in WM. The existence of the domain-general network during WM maintenance supports the argument that WM storage is a unitary system in which visual and auditory items are 
maintained by the same mechanism (Cowan, 1995; Cowan et al., 2011; Majerus et al., 2010).

\section{PART III. NONVERBAL AUDITORY WORKING MEMORY}

In Parts I and II, two analyses were performed on a visual and auditory WM experiment. The results showed that (1) the left anterior IPS does not code domain-specific information in visual and auditory WM, and (2) that a domain-general neural network, which includes the left anterior IPS, is responsible for both visual and auditory WM maintenance. Thus, both studies support the domain-general account of WM storage.

These analyses and most previous neuroimaging studies, however, are focused on categorical stimuli, such as visual colors/simple shapes, verbal letters/digits, etc. For these stimuli, categorical representations can be easily formed once the stimuli enter perception. For example, both a red colored square and a spoken word "red" could invoke the categorical representation of "redness". Categorical representations are independent of stimuli domains and are therefore likely to be the representations stored in the domain-general system of WM.

If the domain-general system stores categorical representations, what if the input stimuli are difficult to categorize? Such types of stimuli include, for example, complex shapes/sounds without associations with real world objects, tones that do not resemble the frequency of musical notes, etc. If no categorical information could be extracted from the stimuli, the domain-general system will likely have no representations to keep. In this case, the left anterior IPS probably will not be activated during WM maintenance. 
This issue is investigated using nonverbal sounds in this study. Nonverbal sounds refer to the auditory stimuli that are not associated with any verbal label and are therefore difficult to be categorized. The brain activations underlying nonverbal auditory WM have been investigated in very few studies (Grimault et al., 2009; Rinne et al., 2009; Strand et al., 2008). Even these few studies yielded very different results. Strand et al. (2008) used acoustic pseudo-words with varied numbers of syllables in a changedetection task. They found that the middle temporal gyrus and inferior frontal gyrus were activated during the maintenance period. The IPS, however, was activated during the response but not the maintenance period. Grimault et al. (2009) used varied numbers of tones in two change-detection tasks. One task was done with magnetoencephalography (MEG), and the other was done with fMRI. In both MEG and fMRI experiments, they found significant activation in the superior parietal cortex, the angular/supramarginal gyrus, and the superior temporal gyrus. Rinne et al. (2009) used a pitch n-back task and found significant load-dependent activation in the inferior parietal lobe. Thus, it is unclear whether the posterior parietal cortex, especially the IPS, is responsible for the maintenance of nonverbal sounds.

These previous studies provided insightful results on the brain regions involved in nonverbal auditory WM. However, to investigate WM for non-categorical stimuli, several important factors should be controlled: (1) musical tones should be avoided because they are easier to categorize than tones whose frequencies do not resemble those of musical tones; and (2) to focus on WM, sensory memory should be controlled by using post-stimulus masks. 
In this study, we tried to control these factors and examined the brain activation for non-categorical stimuli. The participants were instructed to remember 2, 3, 4, 5, or 6 nonverbal sounds in a change-detection task. We are interested in the load-dependent brain activations, especially in the IPS.

\section{Method}

Participants. Prior to the fMRI experiment, a behavioral pretest was conducted to screen participants for the fMRI scan. The behavior pretest has the same procedure as the fMRI experiment as described in the next section. Only the participants showing a high overall accuracy rate in the behavioral pretest were recruited in the fMRI experiment. The screening criteria was set as an overall accuracy rate of .70 and above, the average accurate rate in a previous behavioral experiment with the same procedure (Experiment 3 in Li, Cowan, \& Saults, 2013).

Seventeen participants (seven male) were recruited in the fMRI study. One participant did not finish the scan due to discomfort in the scanner. Another participant had excessive head movements and was excluded in the analysis. The resulting number of participants was therefore fifteen (seven male, 18-22 years old). The participants were native English speaker, right handed, with normal vision and hearing, and without special music trainings. Prior to scanning, each participant went through a questionnaire to exclude the individuals with potential risks for MRI scan, such as metal in the body, claustrophobia, etc. 
Stimuli, Procedures and timing. The stimuli used in both the behavioral pretest and the fMRI experiment were twelve nonverbal sounds, generated with GarageBand (Apple Inc., Cupertino, California), a program in the Macintosh Operating System, each played by a distinct instrument (Trumpet Section, Smooth Clav, Classic Rock Organ, Negril Bass, Tenor Sax, Space Harpsichord, Grand Piano, Live Pop Horns, Aurora Bell, Pop Flute, Hollywood Strings, and Clean Electric Guitar). The fundamental frequencies of these sound files were then varied in the range of $200 \mathrm{~Hz}$ to $3900 \mathrm{~Hz}$ with a $31 \%$ difference between each two adjacent frequencies. Thus, each sound had a distinct timbre and frequency combination.

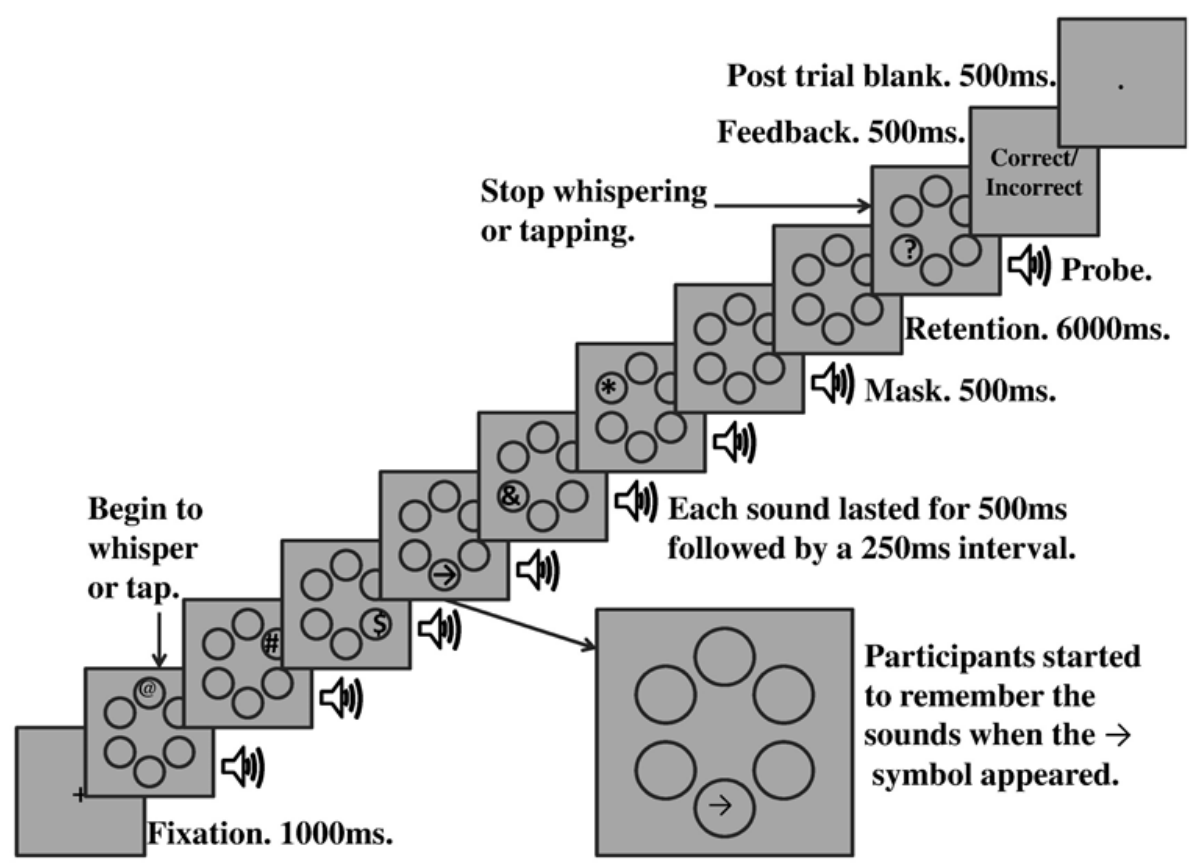

Figure 7. Experimental procedure of the nonverbal auditory WM study. 
The participants performed an auditory WM task as illustrated by Figure 7 . The stimuli were presented with the E-Prime 2.0 software. At the beginning of each trial, a "+" appeared on the center of the screen for $1000 \mathrm{~ms}$, which indicated the onset of a trial and provided a fixation point for the participants. The participants were instructed to start whispering "the" twice a second as soon as they see the "+", and to keep whispering throughout the trial until it was time to respond with a button press. After the "+", six circles were presented on the center of the screen. Six nonverbal sounds, randomly selected from the twelve sounds, were sequentially presented through a headphone, each lasting for $500 \mathrm{~ms}$ with $250 \mathrm{~ms}$ interval between adjacent sounds. A printed character (*, $\&, @, \%, \$$, and ->, randomly arranged) was presented concurrently with each sound, and sequentially in the circles, starting from the circle on the top. The character disappeared as soon as its corresponding sound ended. The participants were instructed to start remembering the sounds when they heard the sound accompanied by a forward arrow ($>$ ) character. The location of the forward arrow was arranged such that the memory load was parametrically manipulated to include five levels: $2,3,4,5$, and 6 sounds.

A 500ms mask sound, produced by simultaneous combination of all the twelve sounds, was presented $500 \mathrm{~ms}$ after the sixth sound ends. After a $6000 \mathrm{~ms}$ delay period during which the participants kept maintaining the sounds and whispering "the", a probe sound was presented through the headphone, accompanied by a "?" in a circle on the screen. The participants were instructed to stop whispering "the" upon hearing the probe sound and seeing the "?", and to decide whether the probe sound was the same as the previous sound in the "?" circle or different from any sound that they were required to remember. In half of the trials, the probe sound was the same as the sound at the "?" 
location during the stimulus presentation, and in the other half of the trials, the probe sound was different from any of the six sounds in the stimulus list. The participants were instructed to respond within 3 seconds. Feedback lasting for $500 \mathrm{~ms}$ was provided after the participants made a response. If the participants failed to respond within 3 seconds, feedback saying "response not detected" was presented on the screen. A blank screen lasting for $500 \mathrm{~ms}$ was presented in the end of a trial.

Two additional conditions with a different stimulus display method were included in the experiment. In these two conditions, only 2 or 4 sounds were presented, each accompanied by a character $((*, \&, @, \%, \$$, and $->)$. The first sound was always accompanied by a forward arrow "->”, indicating that the participants always started to remember the sounds from the first one. The procedure of the two additional conditions was otherwise the same as that of the original conditions. In the following text, we denotes the additional conditions as "Partial", as opposed to the original conditions as "AllSix". The specific memory load in each stimulus presentation method condition is referred to as "AllSix-N" or "Partial-N", respectively, with $\mathrm{N}$ representing the memory load. For instance, memory load 4 in the AllSix condition is referred to as "AllSix-4".

Each AllSix trial lasted for 16 seconds. Each Partial-2 trial lasts for 14 seconds, and each Partial-4 trial lasted for 16 seconds. Every two adjacent trials were separated by a random interval, ranging from 0 to 10 seconds. Randomly varying (jittering) the time between successive trials is a standard technique in fast event-related fMRI experiments to increase the efficiency for separating statistically the brain's activity to stimuli versus responses. 
Each run included 14 trials, and each participant performed 16 functional runs. The duration of each run was 5 minutes and 10 seconds. The experiment lasted for approximately 1.5 hours, including the break time between runs.

Data Acquisition. The fMRI data was collected with a 3-Tesla Siemens Trio MRI scanner located in the Brain Imaging Center at the University of Missouri. For each participant, a T1-weighted (MPRAGE) image and a T2-weighted (SPACE) structural image were collected at the beginning of the experiment. A T2*-weighted spin echo EPI sequence $\left(\mathrm{TR}=2000 \mathrm{~ms}, \mathrm{TE}=30 \mathrm{~ms}\right.$, flip angle $=90 \quad$, in-plane resolution $=4 \times 4 \mathrm{~mm}^{2}, 32$ axial slices with $4 \mathrm{~mm}$ thickness) was used for the functional runs when the participants were performing the memory task. Sixteen functional runs were collected for each participant.

Behavioral Data Analysis. The behavioral data was recorded by the E-prime 2.0 software. A one-way ANOVA of the accuracy rate of the AllSix trials with memory load as the within-subject factor was performed to investigate the effect of memory load. The participant's capacity on each level of memory load was calculated with Cowan's $k$ formula (Cowan 2001). Cowan's k formula estimates WM capacity based on performance in a change detection task in which the test item clearly indicates which item in the original memory set is being tested. It assumes that WM capacity is $k$, meaning that $k$ items can fit into WM. When memory load $N>k$, the hit rate, or the proportion of correct detections of a change, can be calculated as hits $=k / N+(1-k / N) g$, and the false 
alarm rate, or the proportion of answering "change" when there is actually no change, can be calculated as false alarms $=(1-k / N) g$, with $g$ being the guessing rate of answering "change" when no WM information is available. Combining these two equations, WM capacity can be estimated as $k=($ hits - false alarms $) N$.

To show whether different stimulus presentation methods induce different performance patterns, we also selected the Partial trials as well as memory loads 2 and 4 of the AllSix trials for a two-way ANOVA analysis with presentation method and WM load as within-subject two factors.

Whole Brain Analysis. The imaging data was processed with Brainvoyager QX 2.3.1 (Brain Innovation, Maastricht, the Netherlands). During preprocessing, the images were corrected for differences in acquisition time of different slices in each volume, corrected for head motion, realigned and normalized into a standard Talairach atlas, and spatially smoothed with a 6mm FWHM Gaussian filter.

The preprocessed data was analyzed using a random effects general linear model method. For each AllSix condition, the stimulus presentation was modeled as a $6 \mathrm{~s}$ boxcar function; for the Part conditions, the stimulus presentation was modeled as a $2 \mathrm{~s}$ or $4 \mathrm{~s}$ boxcar functions for load 2 and 4, respectively. For both AllSix and Partial conditions, the maintenance and probe periods were modeled as $4 \mathrm{~s}$ and $2 \mathrm{~s}$ boxcar functions, respectively. To minimize the carryover signal from stimulus presentation, the $4 \mathrm{~s}$ boxcar functions modeling the maintenance period were selected to cover the last 4 seconds of 
the maintenance period. Each boxcar function was convolved with a canonical hemodynamic response function.

The boxcar functions were entered into a random effects general linear model. To further reduce artifacts from head movements and scanner signal drifts, six head motion functions and five discrete cosine transform functions for each participant were included in the design matrix.

The behavioral results showed that WM capacity, indexed by $k$ values, increased monotonically from memory load 2 to 6 (See Behavioral Results). To identify brain regions whose activity tracked WM capacity, we first examined high versus low memory contrasts for any two adjacent memory loads in the AllSix trials. This analysis, however, did not reveal any common brain region across all contrasts, suggesting that there may not be a single region responsible for WM storage across all memory loads in this experiment. Therefore, an examination of specific contrasts was necessary to reveal the differences among activation patterns across memory loads.

For the current analysis, we focused on two contrasts: AllSix-6 versus AllSix-4, and AllSix-4 versus AllSix-2, both during the maintenance period. The loads AllSix-2 and AllSix- 6 were selected because they represented the most extreme load conditions in this study, and the load AllSix-4 was selected because it provided a balanced middle point for the AllSix-2 and AllSix-6 conditions.

Additionally, to assess the influence of different stimulus presentation methods, we contrasted the Partial-4 condition versus the Partial-2 condition during the maintenance period and compared the results from the results of the AllSix- 4 versus 
AllSix-2 contrast. To investigate the common activations across these two stimulus presentation methods, a conjunction analysis of both the AllSix-4 versus AllSix-2 and the Partial-4 versus Partial-2 contrasts during the maintenance period was also performed.

In all analyses, multiple comparisons were corrected using cluster level thresholding implemented in BrainVoyager (Goebel et al., 2006; Forman et al., 1995). Unless otherwise specified, an initial threshold was set as $\mathrm{p}=.005$, and the cluster size threshold against a 5\% cluster-wise false discovery rate was determined by 1000 iterations of a Monte Carlo simulation.

Regions of Interest Analysis. The results from Parts I and II suggest that a region in the left anterior IPS is critical for the maintenance of both visual and auditory stimuli. For this analysis, we selected the left anterior IPS region as a region of interest (ROI) and examined its activations at each memory load levels. The left anterior IPS ROI was selected as having the same spatial location and layout as the left IPS region found in the conjunction analysis in Cowan et al. (2011) (Talairach coordinates -27, -46, 31; see Figure 3 in Cowan et al., 2011). Given that processing of tones is often right lateralized, we are also interested in the activation in the right IPS. Therefore, a right anterior IPS ROI was defined as the mirror of the left anterior IPS ROI in the right hemisphere. For both ROIs, the beta values of the AllSix trials during the maintenance period derived from the whole-brain analysis were entered into a one-way ANOVA analysis, which had one factor (memory load) with five levels (memory loads $2,3,4,5$, and 6). 


\section{Results}
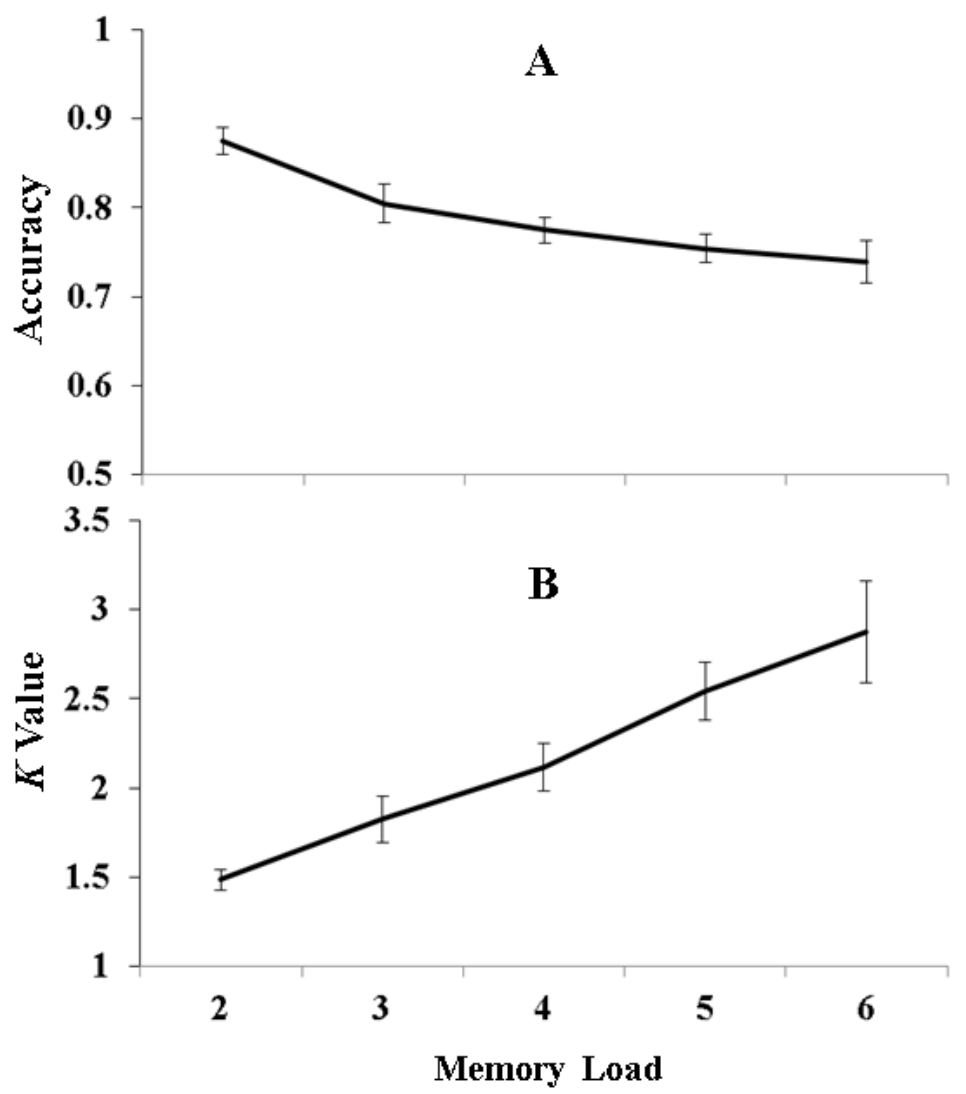

Figure 8. Behavioral results of the AllSix trials in the nonverbal auditory WM experiment. A: average accuracy. B: average $K$ values. $\mathrm{X}$ axis denotes memory loads 2 to 6 . The error bars denotes standard errors.

Behavioral Results. A one-way ANOVA of the accuracy rate in the AllSix trials with memory load as the within-subject factor revealed a significant main effect of memory load, $\mathrm{F}(4,56)=10.39, \mathrm{p}<.001, \eta_{\mathrm{p}}{ }^{2}=.43$. The accuracy rate decreased monotonically with memory load (Figure 8A).

Estimates of WM capacity were calculated with Cowan's $k$ formula (Cowan, 2001). The mean capacity estimates were $1.48,1.83,2.12,2.54$, and 2.88 , for memory load 2, 3, 4, 5, and 6, respectively. It is thus obvious that WM capacity increased monotonically with memory load (Figure 8B). This pattern is different from many 
previous WM studies showing a plateau of WM capacity at set size 3-4 for categorical stimuli (Cowan, 2001; Todd \& Marois, 2004).

To examine the impact of different stimulus presentation methods, a two-way ANOVA with presentation method (AllSix and Partial) and memory load (2 and 4) was performed. Only a main effect of memory load was found, $\mathrm{F}(1,14)=45.93, \mathrm{p}<.001, \eta_{\mathrm{p}}{ }^{2}$ $=.77$. Different stimulus presentation methods thus did not appear to have an impact on behavioral performance. 


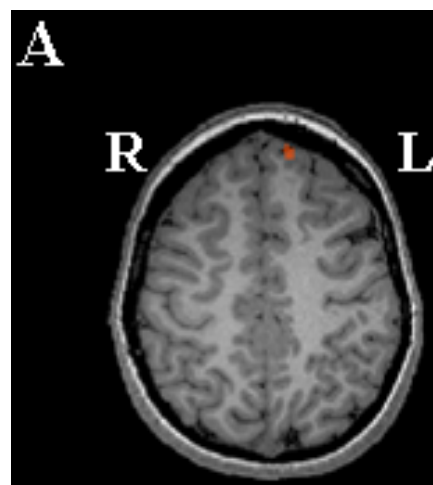

$$
\mathrm{Z}=\mathbf{4 0}
$$

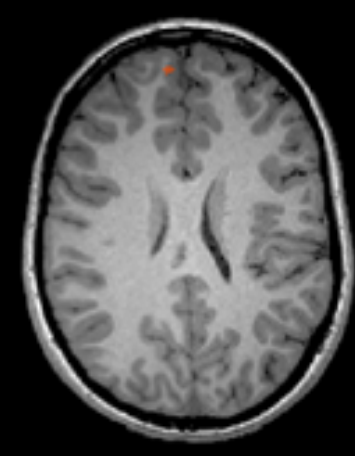

$\mathrm{Z}=\mathbf{2 1}$

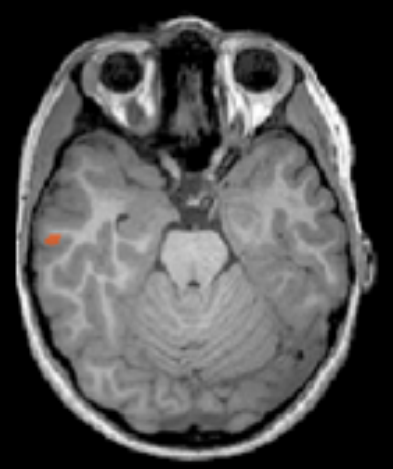

$Z=-18$

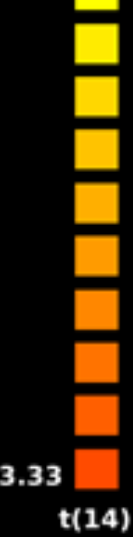

$\mathbf{B}$
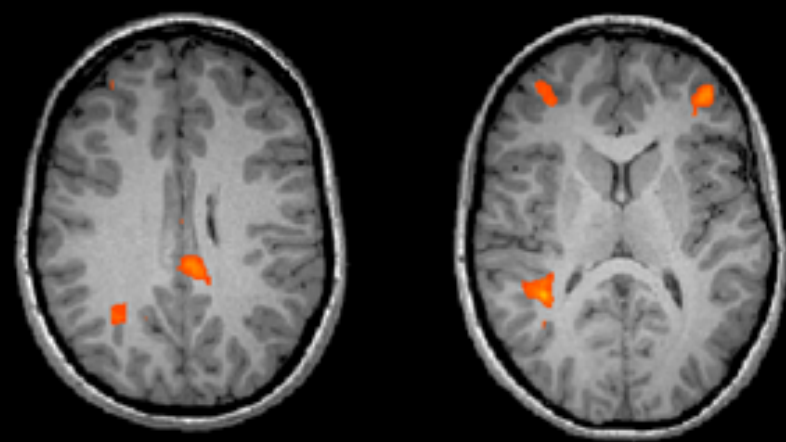

$\mathrm{Z}=\mathbf{1 0}$

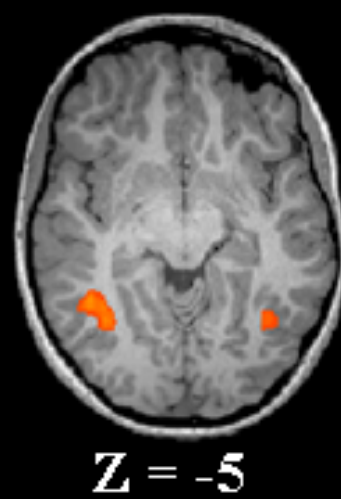

8.00

$$
\mathrm{Z}=\mathbf{2 5}
$$

$$
Z=10
$$$$
\mathrm{Z}=\mathbf{- 5}
$$
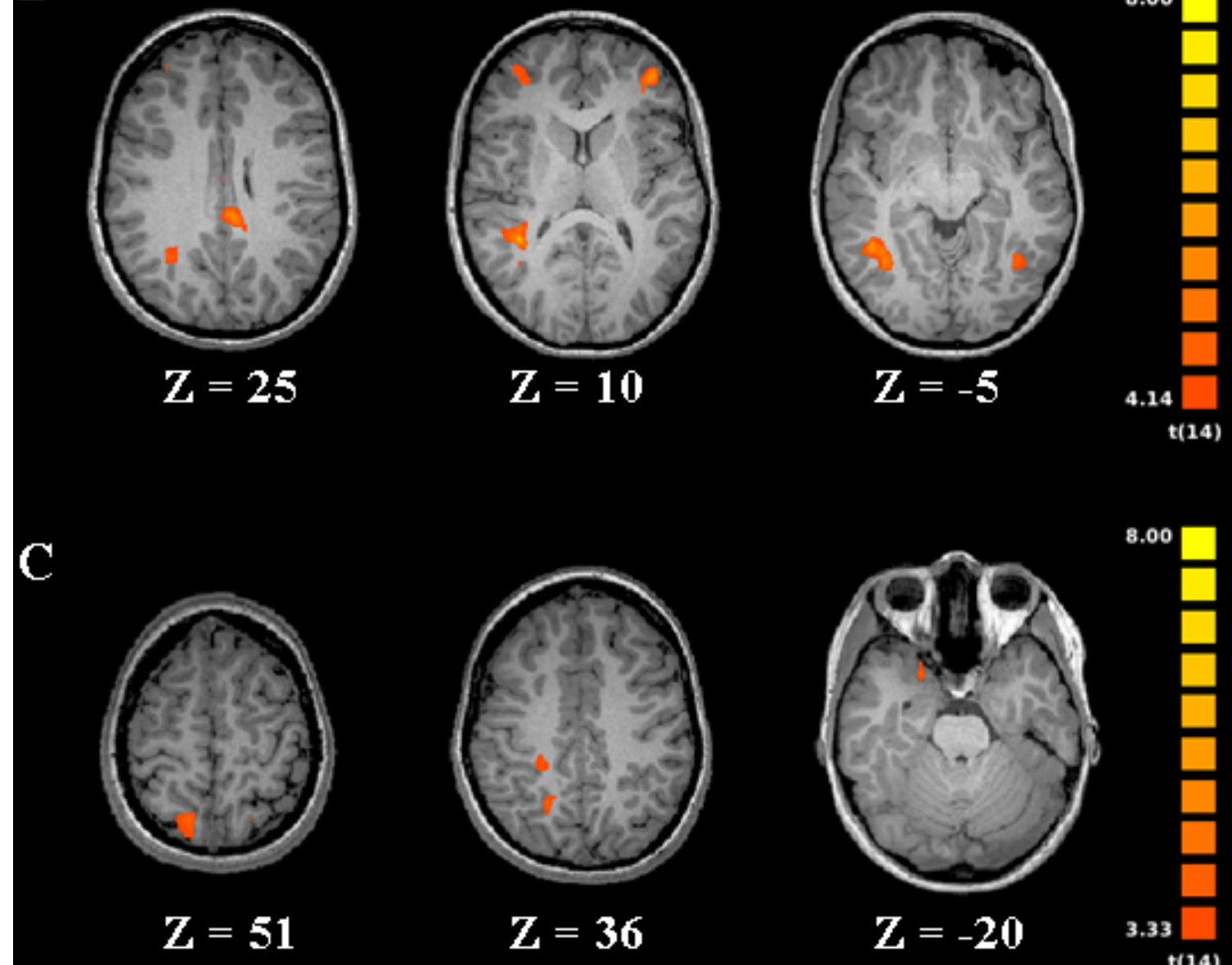

$\mathrm{Z}=\mathbf{- 2 0}$

8.00

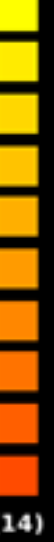

Figure 9. FMRI results of the nonverbal auditory WM study. A: AllSix-4 versus AllSix-2. B: AllSix-6 versus AllSix-4. C: Partial-4 versus Partial-2. All maps follow the radiological convention that the left side of the image denotes the right hemisphere. Maps A and C used an initial statistical threshold of $\mathrm{p}<.005$, 
and map B used an initial statistical threshold of $\mathrm{p}<.001$. All maps were corrected for multiple comparisons using cluster level thresholding implemented in BrainVoyager. Only positive clusters are presented.

Whole Brain Results. The one-way ANOVA of the maintenance period of the AllSix conditions revealed several brain regions responding to memory loads: the left dorsolateral prefrontal cortex, the anterior cingulate cortex, the right rostromedial prefrontal cortex, the right cuneus, and the superior temporal cortex.

Interestingly, the result of the Partial-4 versus Partial-2 contrast was very different from that of the AllSix-4 versus AllSix-2 contrast. The AllSix-4 versus AllSix-2 contrast revealed three significant positive clusters: bilateral rostromedial prefrontal cortex and right inferior temporal gyrus (Figure 9A). The Partial-4 versus Partial-2 contrast showed several significant positive clusters including right precuneus and right inferior frontal gyrus (Figure 9C). The conjunction analysis of the AllSix-4 versus AllSix2 and Partial-4 versus Partial-2 contrasts, however, did not reveal any brain activation, even under a low statistical threshold of $\mathrm{p}<.05$, uncorrected.

Region of Interest Results. The left anterior IPS was analyzed in the ROI analysis. The one-way ANOVA showed a significant main effect of memory load, F(4, $56)=3.20, p<.05$. An inspection of the cell means suggested that the main effect was driven by significantly lower values of memory loads 4 and 5 compared those of memory load 3 (Figure 10). 
Results of the right anterior IPS showed a similar pattern. The one-way ANOVA also showed a significant main effect of memory load, $F(4,56)=2.71, \mathrm{p}<.05$. The main effect was driven by significant lower values of memory load 4 than those of memory loads 3 and 6.

Taken together the results of left and right anterior IPS ROIs, it appears that these regions did not show any load-dependent activation across memory loads. Instead, both regions showed a sharp drop at memory load 4 . Figure 10 shows the time course of percent signal change in the left anterior ROI.

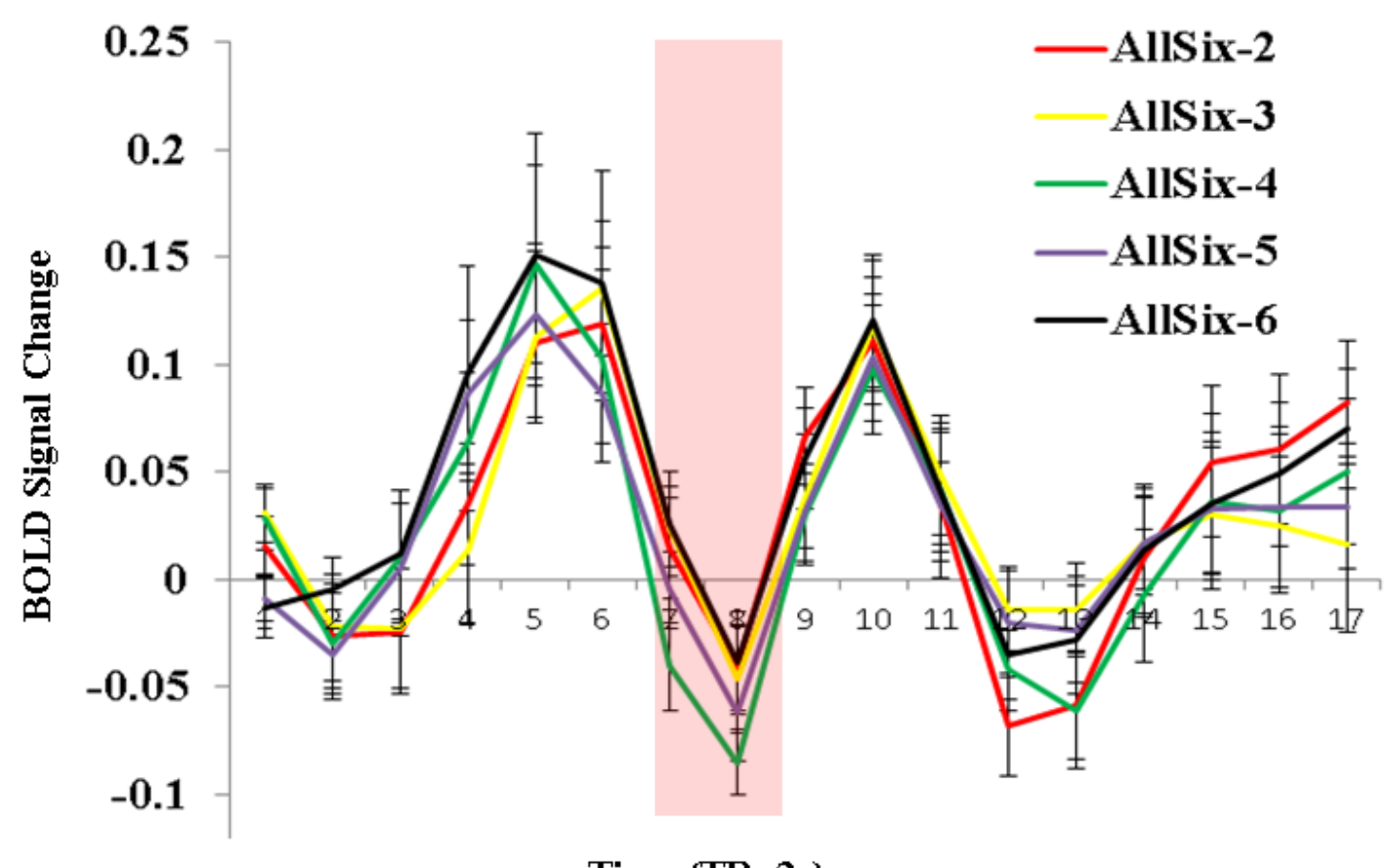

Time $(\mathrm{TR}=2 \mathrm{~s})$

Figure 10. Time course of the average BOLD signal change in the left anterior IPS ROI. Each curve denotes the time course of a memory condition. Red: AllSix-2. Yellow: AllSix-3. Green: AllSix-4. Purple: AllSix-5. Black: AllSix-6. The transparent red rectangle covers the maintenance period.

\section{Discussion}


In this study, we investigated the brain activations underlying a nonverbal auditory WM task. The participants remembered 2, 3, 4, 5, or 6 nonverbal sounds in a change-detection WM task. The behavioral results showed that WM capacity increased monotonically with memory load. The analysis of neuroimaging data, however, did not reveal any brain region showing monotonically increasing activation with memory load, indicating a more complex activation pattern across memory loads. Examination of specific high-versuslow memory load contrasts showed that the AllSix-4 versus AllSix-2 contrast activated different brain regions from the AllSix- 6 versus AllSix- 4 contrast, suggesting that different strategies were used in the maintenance of low and high memory loads. Finally, an ROI analysis showed that the left anterior IPS, previously found to be important for visual and auditory WM, did not exhibit load-dependent activity across memory loads but rather showed a sharp drop of activation at memory load 4.

The monotonically increasing estimates of WM capacity replicated our previous behavioral study (Experiment 3 in Li et al., 2013) and are different from the estimates of WM capacity for categorical visual and auditory stimuli in two aspects: (1) the latter usually increase with memory load and reach a plateau at set size 3 or 4 , and (2) the plateau is usually $3-5$ items, which is higher than the highest capacity (2.88 at memory load 6) in this study. The capacity difference is even more surprising, given that the participants in this study were selected from the top 50 percentile in the behavioral pretest, and that they have already been familiarized with the stimuli in the behavioral pretest before the fMRI experiment. The unusual pattern of WM capacity estimates in this study might be due to the specific characteristics of the stimuli: the nonverbal sounds used in this study were difficult to categorize and thus probably had to be memorized through 
pure acoustic features (Li et al., 2013). This hypothesis, however, needs more investigation from future empirical research.

The AllSix-4 versus AllSix-2 contrast revealed three significant positive clusters: bilateral rostromedial prefrontal cortex and right inferior temporal gyrus (Figure 9A). The medial prefrontal cortex was reported to have widespread reciprocal structural connections with auditory cortex in the macaque (Barbas et al., 1999). The rostromedial prefrontal cortex has been suggested to code topographical information of the tonality surface (Janata et al., 2002). Increased recruitment of the rostromedial prefrontal cortex from memory load 2 to 4 suggests that the participants relied on tonal information to maintain the sounds. The inferior temporal gyrus belongs to the ventral stream of visual processing, which is associated with object recognition and representation (Ungerleider \& Haxby, 1994). It is possible that the participants tried to associate the sounds with their corresponding visual characters to improve their performance even if the visual characters were irrelevant to the WM task.

The AllSix-6 versus AllSix- 4 contrast showed a large group of higher-order cortical areas, including right precuneus, right temporoparietal junction, bilateral middle frontal gyrus, right precentral gyrus, bilateral posterior cingulate cortex, bilateral inferior temporal gyrus and left insula (Figure 9B). Most of these regions, such as the precuneus, middle frontal gyrus, precentral gyrus, posterior cingulate cortex, and insula, are frequently reported to be activated during WM tasks (Owen et al., 2005). The elevated activation of these regions at memory load six and the absence of activation of the rostromedial prefrontal cortex indicate that at higher memory loads, the participants had to use more complex strategies than just relying on the tonal frequencies of the sounds. 
The varied strategies used in low and high memory loads might be the reason why there was no brain region showing monotonically increasing activation with memory load.

Surprisingly, the conjunction analysis of the AllSix-4 versus AllSix-2 and the Partial-4 versus Partial-2 contrasts did not show any common brain activation. Compared to the AllSix-4 versus AllSix-2 contrast, the Partial-4 versus Partial-2 contrast activated a different set of brain regions including right precuneus and right inferior frontal gyrus (Figure 9C). This result indicates that despite the same level of behavioral performance, the two different stimulus presentation methods elicited different brain responses. It is possible that different stimulus presentation methods encouraged the participants to apply different strategies to maintain the sounds.

An important difference between this study and Parts I and II is that the left anterior IPS did not show a load-dependent activation pattern in this study. Instead, the activations in both left and right anterior IPS remained unchanged at memory loads 2 and 3 and showed a sharp drop at memory load 4 (Figure 10). Combining results from the whole-brain analysis, it is possible that the participants experienced a strategy change at memory load 4. At lower memory loads ( 2 and 3 ), the participants could have relied on the acoustic features of the sounds as evidenced by the activation of the rostromedial prefrontal cortex, whereas at higher memory loads (5 and 6), they could have applied more complex strategies as evidenced by the activation of a large group of association areas. This strategy change might have affected the activation level in the anterior IPS, which is proved to be a central hub connecting different modules in the brain (Crossley et al., 2013). 
Given that the left anterior IPS did not show load-dependent activation, it appears that this region may not be responsible for the storage of nonverbal sounds. A possibility is that the left anterior IPS stores abstract, categorical information, which is difficult to be extracted from the nonverbal sounds in this study. An alternative possibility is that due to various strategies used for different memory loads, the brain activation did not show a consistent pattern across different conditions. This inconsistency of strategies might have blurred the difference of activation level in the IPS across memory loads which might have existed if a consistent strategy had been used. Future work is needed to examine the IPS activation during WM for non-categorical stimuli, when strategy usage is controlled.

In conclusion, this study was focused on the brain activations during a nonverbal auditory WM task. The results showed that at low memory loads, the participants relied more on the tonal properties of the sounds, and that at high memory loads, more higherorder brain regions were recruited. Different stimulus presentation methods also appear to influence the strategies used to maintain the sounds. In contrast to the results from Parts I and II, the IPS activation did not show load-dependent activation across memory loads, which indicates that the domain-general system may not store representations of nonverbal sounds.

\section{GENERAL DISCUSSION}

This dissertation is aimed to investigate an important issue in WM: are stimuli from different sensory domains maintained in domain-specific stores or in a categorical, abstract domain-general store? This issue was studied in three related parts. Parts I and 
II re-analyzed an fMRI data set on visual and auditory WM from a previous study (Experiment 2 in Cowan et al., 2011), and Part III was focused on a new fMRI experiment on nonverbal auditory WM.

In Part I, we applied MVPA to the visual and auditory WM data and found that a region in the left anterior IPS, previously found to be involved in both visual and auditory WM maintenance (Cowan et al., 2011), did not code specific information about stimulus domains. In Part II, we analyzed the neural networks underlying the same data set and found that a region in the left anterior IPS, which was spatially proximal to the IPS regions in Part I, belonged to a neural network showing memory load effect during WM maintenance for both visual and auditory stimuli. Thus, both Parts I and II suggest that the left anterior IPS is responsible for domain-general WM storage of categorical visual and auditory information.

In Part III, we analyzed a nonverbal auditory WM data set and found some evidence that different strategies were used for low and high memory loads and different stimulus presentation methods. In contrast to Parts I and II, however, the left anterior IPS activation did not show load-dependent activation across memory load conditions in Part III. We reasoned that the left anterior IPS might store abstract, categorical information which is difficult to be extracted from the nonverbal sounds used in this experiment. This is compatible with the proposed role of the left anterior IPS as a domain-general region, given that a domain-general region should store abstract representations independent of stimulus domains. 
All three approaches suggest that the left anterior IPS is part of a neural network for the maintenance of domain-general, abstract representations in WM, which supports the domain-general account that stimuli of different domains share a common WM storage system. Our finding appears to conflict some earlier studies, which found dissociable brain activations for WM of different domains (Courtney et al., 1996; Haxby et al., 1994; Smith \& Jonides, 1999; Ungerleider et al., 1998). Smith and Jonides (1999) reviewed neuroimaging studies and found different prefrontal regions for verbal, spatial, and visual WM storage. Specifically, they found that verbal WM storage recruits the left prefrontal cortex, and that spatial and object WM recruit the right prefrontal cortex, which supports the domain-specific account of WM storage. However, their review was focused on the prefrontal lobe, and the parietal lobe activations were presented but not discussed. In fact, verbal, spatial, and non-face object WM reviewed in Smith and Jonides (1999) all recruited spatially close brain regions in the posterior parietal cortex (see Figure 3 and 4 in Smith \& Jonides, 1999).

Several other early studies compared spatial and face object WM and found that spatial WM activates the posterior parietal lobe and the dorsolateral prefrontal cortex, and that face WM activates the inferior temporal lobe and the ventrolateral prefrontal cortex (Courtney et al., 1996; Haxby et al., 1994; Ungerleider et al., 1998 ). Thus, these studies appear to support the domain-specific account that spatial locations and faces do not share a common storage system. The discrepancy between these studies and our finding might be due to specific natures of face processing. Some researchers found that recognition of face, compared to common objects, activates specific regions in the fusiform gyrus (Kanwisher et al., 1997), which are the same as the inferior temporal 
regions reported in the face WM studies. It is thus possible that people tend to rely on specific characteristics instead of abstract representations to remember a face in WM. If little or no abstract information is used in face WM, then the abstract domain-general storage system is probably not recruited, as the case in our Part III study.

Our results in Part III did not show any load-dependent activation in the IPS. This is consistent with Strand et al. (2008), which investigated brain activations in a WM task of pseudo-words with 5, 7, or 9 syllables and did not find load-dependent activation in the IPS. Instead, they found significant load-dependent activations in the auditory cortex and the left inferior frontal gyrus, indicating that rehearsal was used to maintain the pseudo-words. This is consistent with our finding that the rostromedial prefrontal cortex, a region coding topographical information of the tonality surface (Janata et al., 2002), was more active in the AllSix-4 condition than in the AllSix-2 condition. These findings indicate that when rehearsal is the main strategy used in a WM task, the abstract domaingeneral storage system might become unnecessary, and the IPS, therefore, might not be recruited.

Taken together the above discussions, it appears that the domain-general storage system might not be recruited in all types of WM tasks. The term "domain-general" does not necessarily apply to every possible stimulus types. Specifically, the domaingeneral storage system does not seem to function when people remember specific characteristics of certain stimuli such as faces, or when people use rehearsal as a primary strategy to maintain phonological/auditory stimuli. We propose that the domain-general system is recruited only when abstract categorical representations are used for WM maintenance, as the case in our Parts I and II studies. 
The existence of an abstract domain-general storage system does not conflict with the existence of domain-specific storage systems for specific domains. In fact, the domain-specific storage systems are necessary to maintain stimulus details, so that people are able to maintain not only an abstract concept but also some fine details of a stimulus. Some neuroimaging studies on visual WM have shown that the posterior visual regions, although not showing elevated activation, coded visual stimulus details in a sub-threshold manner during WM maintenance (Harrison \& Tong, 2009; Lewis-Peacock \& Postle, 2012; Riggall \& Postle, 2012). This result provides evidence that the domain-specific systems are recruited during not only encoding but also maintenance period in WM.

The relative contribution from the domain-general and domain-specific systems to WM storage is complex and may vary depending on stimulus types and the strategies used by the participants. Part III in this dissertation showed that the domain-general system did not seem to be recruited in a nonverbal auditory WM task, but a domainspecific system coding topographical information of the tonality surface was used when memory load was low. In contrast, Parts I and II showed that during the maintenance period in a visual and verbal auditory WM study, the domain-general system was activated, but the domain-specific systems did not show above-threshold elevated activation, which indicates that the domain-general system might have played a more important role in the task used in Parts I and II. It thus seems that the domain-general system is more critical when abstract categorical representations are used to maintain the items in WM.

In conclusion, in this dissertation three approaches were used to address the domain-specific versus domain-general argument regarding WM storage. The results 
show that the left anterior IPS belongs to a neural network for the maintenance of both categorical visual and auditory WM. When non-categorical auditory stimuli were used, however, the brain activations showed complex patterns for different memory loads, and the left anterior IPS did not show a load-dependent activation pattern, which confirms that the domain-general system stores abstract, categorical information. Combining all three approaches, this dissertation supports the domain-general account of WM storage. To achieve a comprehensive picture of the brain mechanism for WM storage, future work is needed to investigate the interactions between the domain-general and domain-specific networks, as well as the interactions between the left anterior IPS and the other domaingeneral brain regions. 


\section{REFERENCES}

Abe, M., Hanakawa, T., Takayama, Y., Kuroki, C., Ogawa, S., \& Fukuyama, H. (2007). Functional coupling of human prefrontal and premotor areas during cognitive manipulation. The Journal of Neuroscience, 27, 3429-3438. doi:10.1523/JNEUROSCI.4273-06.2007

Baddeley, A.D. (1986). Working memory. Oxford: Oxford University Press, Clarendon Press.

Baddeley, A. D., \& Hitch, G. J. (1974). Working memory. In G. A. Bower (Ed.), Recent advances in learning and motivation (Vol. 8, pp. 47-90). New York: Academic Press.

Barbas, H., Ghashghaei, H., Dombrowski, S. M., \& Rempel-Clower, N. L. (1999). Medial prefrontal cortices are unified by common connections with superior temporal cortices and distinguished by input from memory-related areas in the rhesus monkey. The Journal of Comparative Neurology, 410, 343-367.

Birn, R. M., Bandettini, P. A., Cox, R. W., Jesmanowicz, A., \& Shaker, R. (2005). Magnetic field changes in the human brain due to swallowing or speaking. Magnetic Resonance in Medicine, 40, 55-60. doi: 10.1002/mrm.1910400108

Bishop, C. M., \& Nabney, I. T. (1996). Netlab Toolbox. Neural Computing Research Group, Aston University, Birmingham, UK.

Boersma, P., \& Weenink, D. (2009). Praat: doing phonetics by computer (Version 5.2.02) [Computer program]. Retrieved Sept, 2010, from http://www.praat.org/

Buckner, R. L., Andrews-Hanna, J. R., \& Schacter, D. L. (2008). The brain's default network: anatomy, function, and relevance to disease. Annals of the New York Academy of Sciences, 1124, 1-38. doi:10.1196/annals.1440.011

Bush, G., Luu, P., \& Posner, M. (2000). Cognitive and emotional influences in anterior cingulate cortex. Trends in Cognitive Sciences, 4, 215-222. doi: 10.1016/S13646613(00)01483-2

Chang, C., Crottaz-Herbette, S., \& Menon, V. (2007). Temporal dynamics of basal ganglia response and connectivity during verbal working memory. NeuroImage, 34, 1253-1269. doi:10.1016/j.neuroimage.2006.08.056

Chein, J. M., Moore, A. B., \& Conway, A. R. A. (2011). Domain-general mechanisms of complex working memory span. NeuroImage, 54, 550-559.

doi:10.1016/j.neuroimage.2010.07.067

Cocchini, G., Logie, R.H., Della Sala, S., MacPherson, S.E., \& Baddeley, A.D. (2002). Concurrent performance of two memory tasks: Evidence for domain-specific WM systems. Memory and Cognition, 30, 1086-1095.

Cohen, J., Perlstein, W., \& Braver, T. (1997). Temporal dynamics of brain activation during a working memory task. Nature, 386, 604-608. doi: 10.1038/386604a0

Cohen, J. R., Sreenivasan, K. K., \& D’Esposito, M. (2012). Correspondence between 
stimulus encoding- and maintenance-related neural processes underlies successful working memory. Cerebral Cortex. doi:10.1093/cercor/bhs339

Courtney, S. M., Ungerleider, L. G., Keil, K., \& Haxby, J. V. (1996). Object and spatial visual working memory activate separate neural systems in human cortex. Cerebral cortex, 6, 39-49.

Cowan, N. (1995). Attention and memory: An integrated framework. Oxford, England: Oxford University Press.

Cowan, N. (2001). The magical number 4 in short-term memory: A reconsideration of mental storage capacity. Behavioral and Brain Sciences, 24, 87-185.

Cowan, N., Li, D., Moffitt, A., \& Becker, T. (2011). A neural region of abstract working memory. Journal of Cognitive Neuroscience, 23, 2852-2863. doi: 10.1162/jocn.2011.21625

Cox, R. (1996). AFNI: software for analysis and visualization of functional magnetic resonance neuroimages. Computers and Biomedical Research, 29, 162-173.

Culham, J. C., \& Kanwisher, N. G. (2001). Neuroimaging of cognitive functions in human parietal cortex. Current Opinion in Neurobiology, 11, 157-163. doi: $10.1017 / \mathrm{S} 0140525 \mathrm{X} 01593929$

D’Esposito, M., Cooney, J. W., Gazzaley, A., Gibbs, S. E. B., \& Postle, B. R. (2006). Is the prefrontal cortex necessary for delay task performance? Evidence from lesion and FMRI data. Journal of the International Neuropsychological Society: JINS, 12, 248-260. doi:10.1017/S1355617706060322

Destrieux, C., Fischl, B., Dale, A., \& Halgren, E. (2010). Automatic parcellation of human cortical gyri and sulci using standard anatomical nomenclature. NeuroImage, 53,1-15. doi:10.1016/j.neuroimage.2010.06.010

Edin, F, \& Klingberg, T. (2009). Mechanism for top-down control of working memory capacity. Proceedings of the National Academy of Sciences of the United States of America, 106, 6802-6807. doi: 10.1073/pnas.0901894106

Edin, F., Macoveanu, J., Olesen, P., Tegnér, J., \& Klingberg, T. (2007). Stronger synaptic connectivity as a mechanism behind development of working memory-related brain activity during childhood. Journal of Cognitive Neuroscience, 19, 750-760. doi:10.1162/jocn.2007.19.5.750

Fiebach, C. J., Rissman, J., \& D’Esposito, M. (2006). Modulation of inferotemporal cortex activation during verbal working memory maintenance. Neuron, 51, 251261. doi:10.1016/j.neuron.2006.06.007

Gazzaley, A., Rissman, J., Cooney, J., Rutman, A., Seibert, T., Clapp, W., \& D’Esposito, M. (2007). Functional interactions between prefrontal and visual association cortex contribute to top-down modulation of visual processing. Cerebral Cortex, 17 Suppl 1, i125-i135. doi:10.1093/cercor/bhm113 
Gazzaley, A., Rissman, J., \& D’Esposito, M. (2004). Functional connectivity during working memory maintenance. Cognitive, Affective, \& Behavioral Neuroscience, 4, 580-599.

Goebel, R., Roebroeck, A., Kim, D.S., \& Formisano, E. (2003). Investigating directed cortical interactions in time-resolved fMRI data using vector autoregression modeling and Granger causality mapping. Magnetic Resonance Imaging, 21, 1251-1261.

Grefkes, C., \& Fink, G. R. (2005). The functional organization of the intraparietal sulcus in humans and monkeys. Journal of Anatomy, 207, 3-17. doi:10.1111/j.14697580.2005.00426.x

Grimault, S., Lefebvre, C., Vachon, F., Peretz, I., Zatorre, R., Robitaille, N., \& Jolicoeur, P. (2009). Load-dependent brain activity related to acoustic short-term memory for pitch: magnetoencephalography and fMRI. Annals of the New York Academy of Sciences, 1169, 273-277. doi:10.1111/j.1749-6632.2009.04844.x

Grill-Spector, K., Kourtzi, Z., \& Kanwisher, N. (2001). The lateral occipital complex and its role in object recognition. Vision Research, 41, 1409-1422. doi: 10.1016/S0042-6989(01)00073-6

Habeck, C., Rakitin, B., Steffener, J., \& Stern, Y. (2012). Contrasting visual working memory for verbal and non-verbal material with multivariate analysis of fMRI. Brain Research, 1467, 27-41. doi:10.1016/j.brainres.2012.05.045

Hampson, M., Driesen, N. R., Skudlarski, P., Gore, J. C., \& Constable, R. T. (2006). Brain connectivity related to working memory performance. The Journal of Neuroscience, 26, 13338-13343. doi:10.1523/JNEUROSCI.3408-06.2006

Hampson, M., Driesen, N., Roth, J. K., Gore, J. C., \& Constable, R. T. (2010). Functional connectivity between task-positive and task-negative brain areas and its relation to working memory performance. Magnetic Resonance Imaging, 28, 1051-1057. doi:10.1016/j.mri.2010.03.021

Harrison, S. A, \& Tong, F. (2009). Decoding reveals the contents of visual working memory in early visual areas. Nature, 458, 632-635. doi:10.1038/nature07832

Haxby, J. V, Horwitz, B., Ungerleider, L. G., Maisog, J. M., Pietrini, P., \& Grady, C. L. (1994). The functional organization of human extrastriate cortex: a PET-rCBF study of selective attention to faces and locations. The Journal of Neuroscience, 14, 6336-6353.

Honey, G. D., Fu, C. H. Y., Kim, J., Brammer, M. J., Croudace, T. J., Suckling, J., Pich, E. M., Williams, S. C. R., \& Bullmore, E. T. (2002). Effects of verbal working memory load on corticocortical connectivity modeled by path analysis of functional magnetic resonance imaging data. NeuroImage, 17, 573-582. doi:10.1006/nimg.2002.1193

Hunter, M. A., \& Takane, Y. (2002). Constrained principal component analysis: Various applications. Journal of Educational and Behavioral Statistics, 27, 105-145. doi: 10.3102/10769986027002105 
Janata, P., Birk, J. L., Van Horn, J. D., Leman, M., Tillmann, B., \& Bharucha, J. J. (2002). The cortical topography of tonal structures underlying Western music. Science, 298, 2167-2170. doi:10.1126/science.1076262

Kane, M. J., Hambrick, D. Z., Tuholski, S. W., Wilhelm, O., Payne, T. W., \& Engle, R. W. (2004). The generality of working memory capacity: a latent-variable approach to verbal and visuospatial memory span and reasoning. Journal of Experimental Psychology: General, 133, 189-217. doi:10.1037/00963445.133.2.189

Kim, J. S., Jung, W. H., Kang, D.-H., Park, J.-Y., Jang, J. H., Choi, J.-S., Choi, C.-H., Kim, J., \& Kwon, J. S. (2012). Changes in effective connectivity according to working memory load: an FMRI study of face and location working memory tasks. Psychiatry Investigation, 9, 283-292. doi:10.4306/pi.2012.9.3.283

Koelsch, S., Schulze, K., Sammler, D., Fritz, T., Mueller, K., \& Gruber, O. (2009). Functional architecture of verbal and tonal working memory: an fMRI study. Human Brain Mapping, 30, 859-873.

Kondo, H., Morishita, M., Osaka, N., Osaka, M., Fukuyama, H., \& Shibasaki, H. (2004a). Functional role of the cingulo-frontal network in performance on working memory. Neuroimage, 21, 2-14.

Kondo, H., Osaka, N., \& Osaka, M. (2004b). Cooperation of the anterior cingulate cortex and dorsolateral prefrontal cortex for attention shifting. NeuroImage, 23, 670679. doi:10.1016/j.neuroimage.2004.06.014

Kuo, B.-C., Yeh, Y.-Y., Chen, A. J.-W., \& D’Esposito, M. (2011). Functional connectivity during top-down modulation of visual short-term memory representations. Neuropsychologia, 49, 1589-1596. doi:10.1016/j.neuropsychologia.2010.12.043

Lancaster, J. L., Tordesillas-Gutiérrez, D., Martinez, M., Salinas, F., Evans, A., Zilles, K., Mazziotta, J. C., \& Fox, P. T. (2007). Bias between MNI and Talairach coordinates analyzed using the ICBM-152 brain template. Human Brain Mapping, 28, 1194-205. doi:10.1002/hbm.20345

Lenartowicz, A., \& McIntosh, A. R. (2005). The role of anterior cingulate cortex in working memory is shaped by functional connectivity. Journal of Cognitive Neuroscience, 17, 1026-1042. doi:10.1162/0898929054475127

Lent, R., Azevedo, F. A. C., Andrade-Moraes, C. H. and Pinto, A. V. O. (2012), How many neurons do you have? Some dogmas of quantitative neuroscience under revision. European Journal of Neuroscience, 35, 1-9. doi: 10.1111/j.14609568.2011.07923.x

Lewis-Peacock, J. A, Drysdale, A. T., Oberauer, K., \& Postle, B. R. (2012). Neural evidence for a distinction between short-term memory and the focus of attention. Journal of Cognitive Neuroscience, 24, 61-79. doi:10.1162/jocn_a_00140

Lewis-Peacock, J. A., \& Postle, B. R. (2012). Decoding the internal focus of attention. Neuropsychologia , 50, 470-478. doi:10.1016/j.neuropsychologia.2011.11.006 
Li, D., Cowan, N., \& Saults, J. S. (2013). Estimating working memory capacity for lists of nonverbal sounds. Attention, Perception \& Psychophysics, 75, 145-160. doi: 10.3758/s13414-012-0383-z

Linden, D. E. J., Oosterhof, N. N., Klein, C., \& Downing, P. E. (2012). Mapping brain activation and information during category-specific visual working memory. Journal of Neurophysiology, 107, 628-639. doi:10.1152/jn.00105.2011

Ma, L., Steinberg, J. L., Hasan, K. M., Narayana, P. A, Kramer, L. A, \& Moeller, F. G. (2012). Working memory load modulation of parieto-frontal connections: Evidence from dynamic causal modeling. Human Brain Mapping, 33, 1850-1867. doi:10.1002/hbm.21329

Majerus, S., D’Argembeau, A., Perez, T.M., Belayachi, S., Van der Linden, M., Collette, F., Salmon, E., Seurinck, R., Fias, W., \& Maquet, P. (2010). The commonality of neural networks for verbal and visual short-term memory. Journal of Cognitive Neuroscience, 22, 1-24.

Mayer, J., \& Roebroeck, A. (2009). Specialization in the default mode: Task-induced brain deactivations dissociate between visual working memory and attention. Human Brain Mapping, 31,126-139. doi: 10.1002/hbm.20850

Metzak, P. D., Feredoes, E., Takane, Y., Wang, L., Weinstein, S., Cairo, T., Ngan, E .T . C., \& Woodward, T. S. (2011). Constrained principal component analysis reveals functionally connected load-dependent networks involved in multiple stages of working memory. Human Brain Mapping, 32, 856-871. doi: 10.1002/hbm.21072

Metzak, P. D., Riley, J. D., Wang, L., Whitman, J. C., Ngan, E. T. C., \& Woodward, T. S. (2012). Decreased efficiency of task-positive and task-negative networks during working memory in schizophrenia. Schizophrenia Bulletin, 38, 803-813. doi: $10.1093 / \mathrm{schbul} / \mathrm{sbq} 154$

Mitchell, D. J., \& Cusack, R. (2008). Flexible, capacity-limited activity of posterior parietal cortex in perceptual as well as visual short-term memory tasks. Cerebral Cortex, 18, 1788-1798. doi:10.1093/cercor/bhm205

Morey, C.C., \& Cowan, N. (2004). When visual and verbal memories compete: Evidence of cross-domain limits in working memory. Psychonomic Bulletin \& Review, 11, 296-301.

Morey, C.C., \& Cowan, N. (2005). When do visual and verbal memories conflict? The importance of working-memory load and retrieval. Journal of Experimental Psychology: Learning, Memory, and Cognition, 31, 703-713.

Nixon, P., Lazarova, J., Hodinott-Hill, I., Gough, P., \& Passingham, R. (2004). The inferior frontal gyrus and phonological processing: an investigation using rTMS. Journal of Cognitive Neuroscience, 16, 289-300. doi:10.1162/089892904322984571

Norman, K. A, Polyn, S. M., Detre, G. J., \& Haxby, J. V. (2006). Beyond mind-reading: multi-voxel pattern analysis of fMRI data. Trends in Cognitive Sciences, 10, 42430. doi:10.1016/j.tics.2006.07.005 
Owen, A. M., McMillan, K. M., Laird, A. R., \& Bullmore, E. (2005). N-back working memory paradigm: a meta-analysis of normative functional neuroimaging studies. Human Brain Mapping, 25, 46-59. doi:10.1002/hbm.20131

Palva, J. M., Monto, S., Kulashekhar, S., \& Palva, S. (2010). Neuronal synchrony reveals working memory networks and predicts individual memory capacity. Proceedings of the National Academy of Sciences of the United States of America, 107, 75807585. doi:10.1073/pnas.0913113107

Payne, L., \& Kounios, J. (2009). Coherent oscillatory networks supporting short-term memory retention. Brain Research, 1247, 126-32. doi:10.1016/j.brainres.2008.09.095

Pessoa, L., Gutierrez, E., Bandettini, P., \& Ungerleider, L. (2002). Neural correlates of visual working memory: fMRI amplitude predicts task performance. Neuron, 35, 975-987.

Polyn, S. M., Natu, V. S., Cohen, J. D., \& Norman, K. A. (2005). Category-specific cortical activity precedes retrieval during memory search. Science, 310, 19631966. doi:10.1126/science. 1117645

Postle, B.R., Ferrarelli, F., Hamidi, M., Feredoes, E., Massimini, Peterson, M., Alexander, A., \& Tononi, G. (2006). Repetitive transcranial magnetic stimulation dissociates working memory manipulation from retention functions in prefrontal, but not posterior parietal, cortex. Journal of Cognitive Neuroscience, 18, 17121722 .

Riggall, A. C., \& Postle, B. R. (2012). The relationship between working memory storage and elevated activity as measured with functional magnetic resonance imaging. The Journal of neuroscience, 32, 12990-12998. doi:10.1523/JNEUROSCI.189212.2012

Rinne, T., Koistinen, S., Salonen, O., \& Alho, K. (2009). Task-dependent activations of human auditory cortex during pitch discrimination and pitch memory tasks. The Journal of Neuroscience, 29, 13338-13343. doi:10.1523/jneurosci.3012-09.2009

Rissman, J., Gazzaley, A., \& D’Esposito, M. (2008). Dynamic adjustments in prefrontal, hippocampal, and inferior temporal interactions with increasing visual working memory load. Cerebral Cortex, 18, 1618-1629.

Rottschy, C., Langner, R., Dogan, I., Reetz, K., Laird, A. R., Schulz, J. B., Fox, P.T., Eickhoff, S. B. (2012). Modelling neural correlates of working memory: a coordinate-based meta-analysis. NeuroImage, 60, 830-46. doi:10.1016/j.neuroimage.2011.11.050

Saults, J. S., \& Cowan, N. (2007). A central capacity limit to the simultaneous storage of visual and auditory arrays in working memory. Journal of Experimental Psychology: General, 136, 663-684.

Schlösser, R. G. M., Wagner, G., \& Sauer, H. (2006). Assessing the working memory network: Studies with functional magnetic resonance imaging and structural equation modeling. Neuroscience, 139, 91-103. doi:10.1016/j.neuroscience.2005.06.037 
Smith, E. E., Jonides, J. (1999). Storage and executive processes in the frontal lobes. Science, 283, 1657-1661. doi:10.1126/science.283.5408.1657

Strand, F., Forssberg, H., Klingberg, T., \& Norrelgen, F. (2008). Phonological working memory with auditory presentation of pseudo-words - an event related fMRI Study. Brain research, 1212, 48-54. doi:10.1016/j.brainres.2008.02.097

Sundermann, B., \& Pfleiderer, B. (2012). Functional connectivity profile of the human inferior frontal junction: involvement in a cognitive control network. $B M C$ Neuroscience, 13, 119. doi:10.1186/1471-2202-13-119

Takane, Y., \& Hunter, M. A. (2001). Constrained principal component analysis: A comprehensive theory. Applicable Algebra in Engineering, Communication and Computing, 12, 391-419. doi: 10.1007/s002000100081

Tamber-Rosenau, B. J., Dux, P. E., Tombu, M. N., Asplund, C. L., \& Marois, R. (2013). Amodal processing in human prefrontal cortex. Journal of Neuroscience, 33, 11573-11587. doi:10.1523/jneurosci.4601-12.2013

Todd, J.J., \& Marois, R. (2004). Capacity limit of visual short-term memory in human posterior parietal cortex. Nature, 428, 751-754.

Uddin, L. Q., Supekar, K., Amin, H., Rykhlevskaia, E., Nguyen, D. A, Greicius, M. D., \& Menon, V. (2010). Dissociable connectivity within human angular gyrus and intraparietal sulcus: evidence from functional and structural connectivity. Cerebral Cortex, 20, 2636-2646. doi:10.1093/cercor/bhq011

Ungerleider, L. G., Courtney, S. M., \& Haxby, J. V. (1998). A neural system for human visual working memory. Proceedings of the National Academy of Sciences of the United States of America, 95, 883-890.

Ungerleider, L., \& Haxby, J. (1994). "What"and "where"in the human brain. Current Opinion in Neurobiology, 4, 157-165.

Woodman, G. F., \& Vogel, E. K. (2005). Fractionating working memory: consolidation and maintenance are independent processes. Psychological Science, 16, 106-113. doi:10.1111/j.0956-7976.2005.00790.x

Woodward, T. S., Cairo, T. A., Ruff, C. C., Takane, Y., Hunter, M. A., \& Ngan, E. T. C. (2006). Functional connectivity reveals load dependent neural systems underlying encoding and maintenance in verbal working memory. Neuroscience, 139, 317325. doi: 10.1016/j.neuroscience.2005.05.043

Woodward, T., Feredoes, E., \& Metzak, P. (2013). Epoch-specific functional networks involved in working memory. NeuroImage, 65, 529-539. doi: 10.1016/j.neuroimage.2012.09.070

Xu, Y., \& Chun, M.M. (2006). Dissociable neural mechanisms supporting visual shortterm memory for objects. Nature, 440, 91-95.

Yetkin, F. Z., Haughton, V. M., Cox, R. W., Hyde, J., Birn, R. M., Wong, E. C., \& Prost, R. (1996). Effect of motion outside the field of view on functional MR. American Journal of Neuroradiology, 17, 1005-1009. 


\section{APPENDIX \\ Illustration of Constrained Principal Component Analysis}

The purpose of this appendix is to provide an illustration of constrained principal component analysis (CPCA) as applied to a simplified data set.

\section{Simplified Data Set}

For illustration purpose, we suppose that the data set consists of one participant with 7 volumes and 4 voxels. We also suppose that there are only two conditions, two auditory letters $(2 \mathrm{~A})$ and two visual squares $(2 \mathrm{~V})$, and that each condition can be modeled by three time points, with time point 1 representing the encoding period, time point 2 representing the maintenance period, and time point 3 representing the response period. Finally, we suppose that the first three and the last three volumes form a trial of the $2 \mathrm{~A}$ and the $2 \mathrm{~V}$ condition, respectively, and that the fourth volume is inter-trial-interval. To simplify the illustration, hemodynamic delay is not considered.

\section{Multivariate Regression}

Two matrices, $\mathrm{Z}$ and $\mathrm{G}$, are prepared for the linear regression. The matrix $\mathrm{Z}$ consists of the signal from each voxel and each volume. In the simplified data set, $\mathrm{Z}$ is represented as: 
in which sij denotes the signal from volume $\mathrm{i}$ and voxel $\mathrm{j}$.

The matrix $\mathrm{G}$ consists of timing of the experimental conditions. In the simplified data set, $\mathrm{G}$ is represented as:

Condition $2 \mathrm{~A} \quad 2 \mathrm{~V}$

Time Point $\quad 1 \quad 2 \quad 3 \quad 1 \quad 2 \quad 3$

in which each row represents a volume, and each column represents a condition-specific time point. The number "1" means that the volume was collected at the specific time point and thus contains the brain activity during that time point. The number " 0 " means that the volume was not collected at the specific time point.

A multivariate linear regression is performed:

$Z=G C+E$. 
The matrix E consists of errors and represents the variance unrelated to the experimental task. The matrix $\mathrm{C}$ includes regression weights for each experimental condition and each voxel.

Principal Component Analysis (PCA)

Following the linear regression, a PCA is applied to the matrix GC, which represents the task-related variance. Generalized singular value decomposition is applied on GC:

$U D V^{\prime}=G C$

$\mathrm{D}$ is a diagonal matrix with nonnegative real numbers, known as singular values, on the diagonal. Each singular value reflects the amount of variance explained by the corresponding principal component. The number of the singular values is determined by visual inspection of the scree plot which shows the proportion of variance explained by each principal component. In this study, the D matrix included four singular values:

in which di denotes the ith singular value.

The V matrix contains the right-singular vectors. Each column of the V matrix represents a functional network and could be mapped on a brain template to show the involved brain regions. In the simplified data set, $\mathrm{V}$ is represented as: 
in which each row denotes a voxel, and each column denotes a principal component that represents a functional network. The value vij denotes the loading value of voxel $\mathrm{i}$ on component $\mathrm{j}$, which represents the relative importance of voxel $\mathrm{i}$ in component $\mathrm{j}$.

The G matrix contains the left-singular vectors and is used to calculate the condition-specific predictor weights, which is stored in the P matrix:

$U=G P$.

The condition-specific predictor weights in the matrix P represent the contribution of each condition to each principal components for each participant, and thus allow for statistical tests of the effects of WM load condition and time points on the functional networks represented by the principal components. In the simplified data set, $\mathrm{P}$ is represented as:

in which each row denotes a condition-specific time point, and each column denotes a

principal component. The value denotes the contribution of the ith time point 
of the $2 \mathrm{~A}(2 \mathrm{~V})$ condition to component $\mathrm{j}$, and can be used to statistically test the effects of WM load condition and time points on the principal components. 
VITA

Dawei Li was born in China on December, $12^{\text {th }}, 1983$. He received his B.S. degree in Physics from the Peking University in 2007, and his M.A. and Ph.D. degree in Psychology from the University of Missouri in 2013 under the supervision of Dr. Nelson Cowan. He is currently a postdoctoral researcher at Drs. Kevin LaBar and David Rubin's labs at Duke University. His research is focused on applying neuroimaging methods on various aspects of human cognition and emotion. 